
THE NORTH CAROLINA

College of A griculture and Mechanic Arts AGRICULTURAL EXPERIMENT STATION

W. A. WITHERS. A.M., ACTING DIRECTOR

\section{The Flora of North Carolina FROM}

\section{Ranunculaceae to Salviniaceae}

C. W. HYAMS

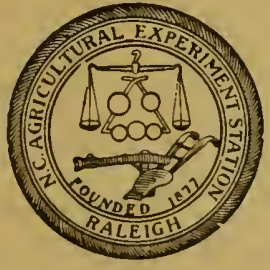

RALEIGH, N. C.

512 


\author{
RALEIGH, N. C.
}

\title{
BOARD OF TRUSTEES
}

W. S. Primpose, Raleigh. President of the Board.

A. LEAzAR, Mooresville.

H. E. Frres, Salem.

D. A. ToMpkins. Charlotte.

T. B. TwITTY, Rutherfordton.

Fr.A.T WOOD. Edenton.

J. C. L. HAPris Raleigh.

L. C. EDTARDS. Oxford.

Jxo. W. HARDEx. JR. Paleigh.

B. E. Bositz Wilmington.

Matt MoORe. Kenansville.

Alex. Q. Holladay. President of the College.

\section{EXPERIMENT STATION STAFF.}

Alex. Q. HolladaY, LL.D., President of the College.*

W. A. Withers. A.M.. Acting Director and Chemist.*

F. E. EMERT, M.S. Agriculturist.*

W. F. MASSET. C.E.. Horticulturist.*

CoOper Certice, D. Y.S.. M.D. (1) Veterinarian.*

G. S. FP.APS. Ph.D.. Assistant Chemist.

J. A. Bizzell. B.S.. Assistant Chemist.

H. W. Primrose, B.S.. Assistant Chemist.

Alex. Rhodes Assistant Horticulturist.

C. W. HYAYs, Assistant Botanist

J. M. Joussos, M.S. Assistant Agriculturist.

B. S. SkLiver, Farm Superintendent.

J. M. Fix, Secretary.

C. M. HCghes. B.E. Clerk.

MPS. L. T. DARBY, Stenographer.

FERTILIZER CONTROL DIVISION.

A. W. BlaIr. A.M., State Chemist.

C. B. WilliaMs, M.S., Assistant Chemist.

C. D. Harris. B.S., Assistant Chemist.

F. G. Kelly, Assistant Chemist.

W. G. HAYwOOD, B.LITT, Assistant Chemist.

H. E. Krvg. Chief Clerk.

Miss M. S. Birdsoxg. Stenographer.

The Director's office is in the main building of the College. Telephone No. $135 \mathrm{C}$. The street cars pass within one hundred yards of the College building.

The Station is glad to receire ant inquiries on agricultural subjects. Address all communications to the Agricultural Experiment Station, and not to individuals. Ther will be referred to the members of the Station staff most competent to answer them.

Samples for analysis should be sent to the State Chemist.

- Member of Slation Council

(1) Oa leave of absence. 


\section{LETTER OF TRANSMITTAL.}

The N. C. College of Agriculture and Mechasic Arts.

THB North Carolixa Agrictlteral Expernert STatioN,

Raleigh, N. C., May 18, 1899.

Prof. W. A. Withers, Acting Director.

I herewith submit a revision of the Flora of North Carolina, prepared by my assistant, Mr. Hyams. The rich Flora of the State has nerer been fully catalogued. That of Dr. Curtis being up to the present the only attempt in this line. I believe that the present list will be valued by botanists as an addition to our knowledge of the babits of these plants.

Yours truly,

IV. F. MASSEY, Horticulturist, Botanist and Entomologist. 
In the vear 1567 , Rer. M. A. Curtis published "A Catalogue of the Indigenous and Naturalized Plants of the state," embracing 1,920 species, exclusire of the lower nrders. Since this time a very large number of species bare been added to the list, as mill be shown further 0 n. Such additions have been rublished, at long intervals, by various parties, and botanists bave long desired a complete Flora of the State, and it is the aim of the present work to supply this waut.

The number of species here recogniz-d is 2,655, comprising 154 families. This brings up to June, 1599, it is believed, all well estab. lished species within our area. In the admission of new species I have pursued a conservative course, and have only admitted those that appear to bear the requisite lests of constancy and persistency in their distinguishing characters. As far as possible the popular, local uames have been given, and I have followed the rerised nomenclature of Britton and Brown, only in such cases as seemed well founded in botanical priority. It gives me plessure to submit this work to your consideration, believing it will aid scientists in determining the localities and range of our regetation to a large extent, and as being, perhaps, the largest local list of plants ever published in the South. I have divided the State into botanical districts, and have indicated the same by the following abbreviations E, C, and W, meaning respectively Eastern, Central and Western. Where a species is found in all the districts the letter $A$ is used. A liue running north through Gaston, Alexander and Alleghany counties would divide the Central and Western districts, while one running north through Bladen, Wakeand Granville counties would diride the Eastern and Central districts. I have criticallyexamined all the various publications bearing on the subjeci, and desire to make due acknowledgements to the authors of the same.

Respectfully,

C. W. HYAMS. 


\section{The Flora of North Carolina.}

FROM

RANUNCULACEAE TO SALVINIACEAE, By C. W. HYAMS, AsgistaMt BOTANIBt.

\section{Flowering Plants.}

Class I-Exogeneous Plants.

\section{Order 1.}

RANUNCULACEAE. (CROWFOOT FAMILI.)

Atragene Americana, Sims. Purple Virgin's Bower. W.

Clematis ochroleuca, Ait. Dwarf Clematis. C. and W. ovata, Pursh. Mountain Clematis. W.

Tiorna, L. Leatber Flower. C. and W. crispa, L. Blue Jessamine. E.

-Virgiuiana, L. Virgin's Bower. C. and W.

Addisonii, Brittou. Brown's Clematis. C.

reticulata, Walt. Sinooth Clematis. E.

Catesbyana, Pursh. Hairy Clematis. E.

Axemore quinquefolia, L. Wind Flower. W.

Caroliniana, Walt. Carolina Anemone. W.

Virginiana, L. Tirginia Anemone. C. and IT.

trifolia, L. Mountaiu Anemone W.

Hepatica triloba, Chaix. Liverwort. C. and W.

"Iacutiloba, D. C. Acute-lobed Liverwort. C. and W.

Thalictrum dioicum, L. Early Meadow Rue. W.

Cornuti, L. Tall Meador Rue. A.

clavatum, D. C. Slender Meadow Rue. IV.

macrostylum, Shuttlaw. Meadow Rue. W.

nudicaule, Schwein. Meadow Rue. C.

anemonoides, Michx. E. and C.

coriaceum (Britton) Small. l'hick Meadow Rue W. purpurascens, L. Purple Meadow Rue. W.

Adoxis antua, L. Pheasant's Eye. A.

Trautvetteria palmata, F. \& M. False Bugbane. W.

Raxurculus aquatilis, L. White Crowfoot. C.

parviflorus, L. Small-flowered Crowfoot. E. and C.

alismaefolius, Geyer. Spear-leaved Crowfoot. C.

pusillus, Poir. Dwarf Crowfoot. E. and C.

muricatus, L. Spiny-fruited Crowfoot. E. and C. 
Raxurcules abortivus, L. Smooth Crowfoot. E. and C. micranthus, Nutt. Rock Crowfoot. E. and C. recurvatus, Poir. Rough Crowfoot. E. and C. sceleratus, L. Biting Crowfoot. E. Pennsylvanicus. L. Bristly Crowfoot. C. Purshii, Rich. Pursh's Crowfoot. C. septentrionalis, Poir. Marsh Crowfoct. E. and C. repens, L. Creeping Crowfoot. A. repens, L. nitidus. Crowfoot. E. fascicularis, Musb]. Tufted Crowfoot. C. hispidus, Miclix. Hispid Crowfoot. A. acris, L. Meadow Crowfiot C. palmatus. Ell. Pallmate Crowfoot. E. Allegluaniensis. Britton. Mountain Crowfoot. W. bulbosus, L. Bulb us Crowfoot. A. parrulus, L. Hairy C'rowiont. E delphinifolius, Torr. Yellow Water Crowfoot. E. oblongifolius, Ell. Obiong-leaver Crowfoot. E. Batrachicm trichophyllum (Chaix). Boss. Water Crowfoot. E. Aquilegra Canalensi:, L. Columbine Cromfoot. A.

rulgaris, L. Purple Crowfoot. C.

coccinea, small. Crowfont. C.

Delphisiug azureum. Michx. Blue Lockspur. W. tricorne, Michx. Dwarf Lockspur. W. exaltalum, Ait Tall Lnckspur. W. Consolida, L. Garden Lnckspur A.

Helleborus viridis, L Green Hellehore. IV.

Acositum uncinatum, L. Wild Moukshoord. WV.

reclinatum, A. Gral. Trailing Monkshood. W.

Zasthorhiza apiifolia, LHer. Yellow Root. A. Hrdrastis Canadensis. L. Golden Seal. W. Caltha palustris, L. Marsh Marigold. C. and WV. Isopyou biternatum, T. \& G False Rue Anemone. C. ACtaEa alba (L.), Mill. White Bueberry. W. rubra (Air.) Wild. Red Baneberry. W.

Cimicifuga racemosa, Ell. Rattle Topl. C. and WV. cordifolia, Pursh. Curdate Rattle Top. W. Americana, Michx Mountain Ratile Top. IT. Mrostres minimus, L. Mouse tail. E.

Order 2.

MAGNOLIACEAE. WAGNOLIA FAMILY.

Magrola grandiflora, L. Magnolia. E. rglauca. L. Sweet Bay. E.

- Embrella, Lam. Umbrella Tree. A. Tripeto racuminata, L Curumber Tree. IV. cordata, Michx. Heart-leaved Magnolia. W. 
Magxolia Fraseri, Walt. Long.leaved Magnolia. W. macrnphylla, Michx. Large-leaved Magnolia. W. Liriodendron Tuliplifera, L. Puplar. A.

Order 3.

ANONACEAE. PAPAW FAMIIY.

Asrmisa triloba, Dunal. Papiw. C. and W. parviflora, Duual. Small Papaw. A.

Order 4 .

MENISPERMACEAE. MOONSEED FAMILY.

Mexispermum Canadense, L. Moonseed. A. Cocculus Carolinus, D. C. Red Moonseed. E. and C. Calycocarpus Lyoni, Nutt. Cupseed. C.

Order 5.

BERBERIDACEAE. BARBERRY FAMILY.

Berberis Canadensis, Pursh. Barberry. IV. vulgaris, L. European Barberry. C. and W. Caulophrluum thalictroides, Michx. Blue Cohosh. WV. DipHYlleia cymosa, Michx. Umbrella Leaf. IW. $\checkmark$ Podophyllum peltatum, L. May Apple. A. Jeffersonia diphylla, (L.) Pers. Twin-leaf. IV.

Order 6.

NYMPHAEACEAE. WATER LILY FAMILY.

Cabovba Caroliniana, Gray. Water-shield. E. Brasena peltata, Pursh. Water-target. E. Nelumbium luteum, Willd. Water Chinquepin. E. Nrmphated advena, Soland. Yellow Pond Lily. A. adrena, minnr, Morong. Small Pond Lily. C. sagittaefolia, Walt. Arrow-leaved Pond Lilly. E. odorata, Ait. White Pond Lilly. E. and C.

Order $\mathrm{i}$.

SARRACENIACEAE. PITCHER PLANT FAMILY.

Sarracexia purpurea, L. Purple Pitcher Plant. E. and C. flava, L. Trumpet-leaf. E. and C. rubra Walt. Red-flowered Trumpet-leaf. S. IV. $\checkmark$ variolaris, Michx. Spotted Trumpet-leaf. S. E.

Order S.

DROSERACEAE. SUNDEW FAMILY.

Drosera filiformis, Raf. Three-leaved Sundew. E. 
Urosera longifolia, L Long.leaved Sunder. rotundifolia. L. Round-leared Sundew. E. and C. brevifolia, Pursh. Shnrt-lenred sundew. E and C. Droxaea muscipula, Ell. Fly "Trap. E.

Order :

PAPAVERACEAE. POPI' FAMILY.

Argemone Mexicana, L. Mexican Puppy. E.

Sarguraria Canadensis L. B ood Root. A.

Chelidonicm majus, L. Celandine. C.

Papaver somniferum, L. Opum Poppr. C. and W.

Rhoeas, L. ('uru Pomsy.

dubium. I smonth-fruited Poppy. A.

Argemone, L. Rough-fruited Poppy. E

Guaccicm luteum, Scop. Sea Poppy. E.

Order 10.

FUMARIACEAE. FLMITIRI FAMILI.

Aduma cirrlosa, Raf Climbing Fumitory. W"

Dicextra Cucullaria. Torr. Dutchman's Breeches W.

eximia, Walp Wild Bleeding heart. W.

Canadensis, Turr. Equirrel Cotn. IV.

Corydals glauca, Pursh. Pink Corydalis. W'. favula, Raf Pale Corrdalis. W.

aurea, Willd. Golden Corydalis E

micrauthum (Eugllm). Brittou. Small-flowered Corydalis. E.

Fumaria officinalis, L. Fumitory. E parviflora, Lam. Suall Fumitory. E.

Order 11 .

\section{CRUCIFERAE. MLSTARD FAMILY.}

Nasturtium lanacetifolium, H. \& A. Tansy Cress. E. palustre, D. C. Mar-b Cress. 'E.

lacustre, Gray. Lake Cress. C.

officinale, R. Br. Water Cress. A.

sylvestre, R. Br. Creeping Yellow Cress. E

hispidum, D. C. Hi-pid Ýllow Cress. E.

Armoracia, Fries. Horseradish. C.

Cardamer rbomboidea, D. C. Spring Cress. E.

rotundifolia, D. C. Round-leared Cress. WT.

spathulata, Michx Spatulate-leared Cress W.

hirsuta, L. Bitter Cresz. C.

Ludoviciana, Hook. Virginia Bitter Cress. C.

Clematitis, Shutilw. Mountain Bitter Cress. IV.

Pennsylranica, Muhl. Pennsylvania Bitter Cress. W. 
Cardamise arenicola, Britton. Sand Bitter Cress. E. parviflora, L. Small flowered Bitter Cress. IV. flexuosa, With. Wuod Bitter Cress. W.

Dentaria diphylla, Michx. Pepper Root. W.

laciniata, Muhl. Cut leaver Pepper Root. C. and WV.

heterophylla, Nutt. Slender Pepper Root. IV.

multifida, Muhl. 'Teruate leaved Pepper Root. C.

Arabis lyrata, 1. . Rock Cress. C.

Canadensis, L. Sickle Pod. C. and W.

laevigata, D. C. Smooth Rock Cress. C. and IV.

Thaliana, L. Thale Cress. C.

hirsuta, scop. Hairy Ruck Cress. W.

Burkii, Porter. Burke's Rock Cress. W.

Barbarea vulgaris, R. Br. Yellow Rocket. E. and C.

praecox, R Br. Scurry Grass. C.

stricta, Andrz. Winter Cress. C.

Sisymbrium Thaliana, Gaud. Mouse-ear Cress. E. and C.

canescens, Nutt. Tansy Mustard E. and C.

officinale, Scop. Herlge Mustard. E. and C.

Allaria, Scop. Garlic Mustard. E. and C.

Draba Caroliniana, Walt. Caroline Whitlaw Grass. E. and C. ramosissima, De:o. Branching Whitlaw Grass. IV.

verna, L. Vernal Whitlaw Griss. E and C.

brachycarpa, Nutt. Short-fruited Whitlaw Grass E. and C.

Camelina sativa, Crantz. False Flax. E.

Sexebiera Coronopus, Puir. Wart Cress. E.

didyma. Lesser Wart Cress. A.

Lepidium Tirginicum, L. Wild Peppergrass. A.

campestre, R. Br. Field Peppergrass. C.

Ruderale, L. Roadside Peppergrass. E.

Capsella Bursa-pastoris, Moench. Shepherd's Purse. A.

Cakile maritima, Scop. Sea Kale. E.

Alrssum maritinum, L. Sweet Alyssum. C.

Sixa PIS nigra, L. Black Mustard. A.

alba, L. White Mustard. A.

arrensis, L. Charlock. A.

juncea, L Indian Mustard. C.

Brassica campestris, L. Turnip. A.

Napus, L. Rape. C.

Raphanus sativus. L. Radish. A.

Hesperis matronalis, L. Dame's Rocket. C.

Order 1?.

CAPPARIDACEAE. CAPER FAMIIY.

Gryandropsis pentaphylla, D. C. E

Polanisia graveolens. Raf. Clammy-need. IT.

Cleone spinosa, L. Spider-flower. C. and W. 


\section{Order 13.}

VIOLACEAE. VIOLET FAMILY.

Nior.a palmata, L. Early Blue Violet. A. cucullata, Ait. Hoored Blue Violet. A. villosa, Walt. Wood Blue Violet. E and C. sororia, Willd. Woolly Blue Violet. C. sagittata, Ait. Arrum-leaved Violet. C. orata, Nutt. Ovate Arrow-leaver Violet. C. pedata, L. Bird's-foot Violet. A. rotundifolia. Michx. Round-leaved Violet. W. blanda, Willd. Sweet White Violet. E. and C. blanda amoena. LeConte. LeCunte's Violet C. primulaefolia, L. Primrose-legved Vinlet. E. aud C. lanceolata, L. Lance-leaved Violet. E. and C. hastata, Michx. Halberd-leaved Violet. W. pubescens, Ait. Hairy Yellow Violet. C. and W. scabriuscula (T. \& G.), Schwein. Smooth Yellow Violet. W. Canadensis, L. Canada Violet W. striata, Ait. Striped or Pale Violet ('. Labradorica, Schrauk. American Dog Violet. C. and W. rostrata, Pursh. Long-spurred Violet. IW. tricolor, L. Panss. A. tenella, Muhl. Field Pansy. C. and W. glaberrima. Ging. Gingin's Violet C. tripartita, Ell. Elliott's Violet. C. canina, L. Pale Dog Violet. C. canina multicaulis, T. \& G. Dog Violet. C. Solea concolor, Ging. Green Violet. A.

Order 14.

CISTACEAE. ROCK ROEE FAMILY.

Heliasthemem maju: B. S. P. Hoary Frostweed. A.

Canadense, Michx. Long-branched Frostreed. E. aud C:

corymbozum, Michx Pine-barren Frostweed. E. and $\mathrm{C}$.

Carolinium, Michx. Carolina Frostweed. E. and C. Hrdsosia montana, Nutt. Mountain Heather. W. ericnides. L Bushy Heather. E.

tomentosa, Nutt. Beach Heather. E.

Lechea minor, L. Thrme-leaved Pin-weed. E. and C. racemulosa, Miclıx. Oblung fruited Pin-weed. W. major. Michx. Hairy Pin-reed. E. and C. thymifolia, Pursh. Beach Pinweed. E. tenuifolia, Michx. Narrow-leared Pin-weed. E. and C. Leggettii, Britt aud Hall Leggett's Pin-reed. E. 
Order 15.

PORTULACACEAE. PARSLANE FAMILY.

portulaca oleracea, L. Parslane. A.

grandiflora, Hook. Garden Parslane. C. and W.

pilosa, L. Hairy Parslane. E

Talinum teretifolium, Pursh. Fame Flower. C. and W.

Claytonia Virginica, L. Spring Beauty. E. and C.

Caroliniana, Michx. Carolina Spring Beauty. W.

Sesuvium pentandrum, Ell. See Parslane. E.

portulacastrum, L. See Parslane. E.

Order 16.

CARYOPHYLLACEAE. PINK FAMILY.

Paronychia dichotoma, Nutt. Forking Whitlow-wort. W. argyrocoma, Nutt. Silver Whitlow-wort. W. herniariodes, Nutt. Hairy Whitlow-wort. E.

Anychia dichotoma, Michx. Forked Chickweed. A. capillacea, D. C. Slender Forked Chickweed. W. Agrostemma Githago, L. Corn Cockle. A. Stipulicida setacea, Michx. E. Silene stellata, Ait. Starry Campion. C. and W. ovata, Pursh. Ovate Starry Campion. C. and W. Wirginica, L. Fire Pink. A. rotundifolia, Nutt. Round-leaved Catchfly. C. Caroliniana, Walt. Carolina Pink. E and C. antirrhina, L. Sleepy Pink. A.

Armeria, L. Sweet William Pink. E. and C. noctiflora, L. Night-blooming Catchfly. A.

Lychnis alba, Mill. White Campion. C. -Saponaria officinalis, L. Snapwort. A.

Vaccaria, L. Cow-herb. C.

Dianthus Armeria, L. Deptford Pink. C.

barbatus, L. Bunch Pink. C.

Alsine media, L. Common Chickweed. A.

squarrosa, Feuzl. Barren Sandwort. E.

glabra, Gray. Glabrous Sandwort. W.

Groenlandica, Spreng. Mountain Sandwort. W.

Michauxii, Fenzl. Narrow-leaved Sandwort, C. and W. Arennaria diffusa, Ell. Hairy Sandwort. E.

serphyllifolia, L Thyme-leaved Sandwort. E. and C. rubra, L. Sand Spurry. E.

Spergula arvensis, L. Pine Cheat. C. and W.

Mollugo verticillata, L. Indian Chickweed. C. and W.

SAGina procumbens, L. Procumbent Pearlwort. C. and IV. decumbens, T. \& G. Decumbent Pearlwort. C. alid W. Elliottii, Fenzl. Elliott's Pearlwort. E. and C. 
Steldakia pubera, Michx. Star Chickwera. C. and W. uniflora, Walt. Marsh Chickweed. E.

Cerastily viscosum, L. Monet-ea-Chickweed. A. semidecandrum, L. Small Mnuse ear Chickreed. C. rulgatum, L. Large Mnne ear Chickweed. A. nutans, Raf. Noddiug (hickweed. A. arvense. L. Field Chickweed. A. Tissa marina (L.), Brimon. Sult marsh spurry. E ScLeraxtuts annume. L (ierman Kuntgras. E.

Order 1:.

\section{MAS.VACEAE. MALLOW FAMILY.}

Malra rotundifulia, L. Dwarf Millow. A. sylvestris, L. High Maliow. C. and W. moschata, L Musk Mallow. ('. and W.

Caldirrhoe triangulata, (Lear.) Gray. Poppy Mallow. IY. Napafa dioica, L. Glade Mallow. C.

Sida spinosa, L. Prickiv Sirla. A.

rhombifolia. L. Sida. If

Elliotti, T. \& G. Ellintl: Sida. C. and Tr.

Abutilox Avicennae, Gaert. Indian Mallow. C. and IT. Modiola multifila, Moench. Bristly-fruited Mallow E. Kosteletzkya Turginica, Presl. E.

Hibrsces Moscheutos, L. Swamp Mailow. A. militaris, Car. Halberiltared Mallow. E. aculeatus, Walt. E.

Trionum, L. Tenice Mallow. A.

Order 18.

\section{TILIACEAE. LINDEX FAMILI.}

- Trlia Americana, L. American Linden. W. pubescens, Ait. Hairy Linden. W. beterophylla, Tent. Mountam Linden. W.

Order 19.

HYPERITACEAE. ST. JOHN'S WORT FAMILY.

Ascrrum stans, Michx. St. Peter's mort. A.

bypericnides, L. Si. Andrew's wort. A.

Hypericuir Kalmianmm, L Kalm'st. John's-ment. W. prolificum, L. Sirubby Kalm S St. John's-mort. W. densiforum, Pursh. Bushy Kalm's St. John's-mort. W. galioides, L,tm. B-d straw Si. Joht'sort. W. adpresum. Bart. Creeping S. John's-mort. C. and W. rirgatum, Lam. Virgate St. John's-wort. C. and W. perforatum, L. Common st. John's-mort. A. 
Hepericum maculatum, Walt. Spotted St. Jobn's-wort. C. aud W. graveolens, Buckley. Mnuntain St. Jolnn's-wort. W. inulitum, L. Dwarf St. Juhn's-wort. C. and W. gymnanthum. Engelm. Clasping-leared St. John'swort. C.

Canadense, L. Cantadian St. Jolnn's.mort. W.

Drummondii, T. \& G. Drummond's St. John's- wort. W. Sarothra. Mirhx. Orange-grass. A.

Virginicum, L. Marsh S.. Juhu's-mort. C. and W'. peliolatum, Walt. Largrr Marsh S. John's-rort. E. and C:

aureum, Bart. Mucrunate St. Johns-wort. W. fasciculatum, Lam. Fascicled St. John's-wort. E. aspalathoides, Willd. Branching St. John's-wort. E. ambiguum, Ell. Linfar-leare I St. John's-wort. II. corymbosum, Muhl. Mountain St. John's-mort. W. Nudiflorum, Michx. Fourangled St. John's-wort. E. and $\mathrm{C}$.

Buckleri, r'urtis. Buckley's St John's-wort. W. pilosum, Walt. Duwny St. Jubn's-wort. E. aud C. acutifolium, Ell. Larger Virgate St. John's-rort. C. glomeratum. W.

Elodes campanulata, Pursh. E. peliolata, Pursh. E.

Order 20.

CAMELLIACEAE. CAMELLIA FAMILY.

Gordosia Lasianthus, L. Loblolly-Bay. E.

Steartia Virginica, Cav. E. and C.

pentagyna, L'Her. W.

Order 21.

MELIACEAE. NAHOGAXY FAMILY.

Melia Azederacb, L. Chiva Tree. A.

Order ??.

\section{LINACEAE, FLAX FAMILY.}

Lisum usitatissimum, L. Flax. C. and W. Virginianum, L. Virginia Flax. A. striatum, Walt Swamp Flax. C. sulcatum Rids. Sulcate Flax. C. and W. medium (Plauch.) Britton. Stiff Yellow Flas. E.

Order 23.

OXALIDACEAE. WOOD-SORREL FAMILY.

Oxalis Acetosella, L. White Word-surrel. W. 
Oxalis violacea, L. Violet Wund-sorrel. C. aud W. corniculata, L. Prucumbent Wnod-sorrel. E. and C. filipes, Small. Slendur Yellow Woor-sorrel. C. stricta, L Upright Yellow Wnod-sorrel. A. recurva, Ell. Large flowered Yellow Wood-sorrel, C. cymosa, small. Tall Yellow Whod-sorrel. C. grandis, Small. Great Yelluw Wood-sorrel. W.

\section{Order 24.}

GERANIACEAE. GERANICM FAMILY.

Geranium maculatum, L. Crane's hill Alum-root. C. and W. Caroliuianum, L. Carolina Crane-bill. A. dissectum, I. W.

Robertianum, L. Herb Rohert. C.

Columbinum, L. L'ng.stalked Crane's-bill.

Molle, L. Crane's-bill. IV'.

Erodicm cicutarium, L. Stork's-bill. E. and C.

Order 25.

\section{BALSAMINACEAE BALSAM FAMILY.}

Impatiexs pallida, Nutt. Pale Touch-me-not. W. fulva, Nutt. Spotted Touch-menot. A.

Order 26.

RUTACEAE. REE FAMILY.

Xasthorylum Americanum, Mill Prickly Ash. W.

Clava-Herculis, L. Suthern Prickly Ash. E.

Clara-Herculis Fruticosum, Gray. Shrubby Prickly Ash. E.

$\checkmark$ Ptelia trifoliata, L. Wafer-ash. A.

\section{Order $2 \%$.}

SIMARUBACEAE. AILANTHUS FAMILY.

Alianthes glandulosa, Desf. Tree-of-Haven. A.

Order 28.

ANACARDIACEAE. SUMAC FAMILY.

Ruts copallina. L. Upland Sumac. A.

- typhina, L. Staghorn Sumac C. and W.

glabra, L. Scarlet Sumac. C, and WV. aromatica, Ait. Fragran' Sumac. WW. aromatica inollis, Gray. Fragrant Sumac. C. Vernix, L. Poison Sumac. A. radicaus, $\mathrm{L}$ Poison Iry. A. Toxicodendron, L. Poism Oak. A. pumila, Michx. Hairy Sumac. E and C.

, Cotinoides, Nutt. Smoke-tree. C. 
Order 29.

VITACEAE. VINE FAMILY.

ᄃVitis Labrusca, L. Fox Grape. A.

raestivalis, Michx. Summer Grape. A.

- cordifolia, Michx. Frost Grape. A.

rotundifolia, Michx. Muscadine Grape. A.

- bicolor, LeConte. Winter Grape. A.

Baileyana, Munson. Bailey's Grape. W.

Cissus Ampelopsis, Pers. Simple Ampelopsis. E.

stans, Pers. Pepper-vine. E.

Ampelopsis quinquefolia, Michx. Virginia Creeper.

A.

Order 30.

\section{RHAMNACEAE. BUCKTHORN FAMILY.}

Bercheria volubilis, D. C. Supple Jack. E. and C.

Rhamnus minutiflorus, Michx. Vine-lika Buckthorn. E.

Carolinianus, Walt. Carolina Buckthorn. A.

lanceolata, Pursh. Lance-leaved Buckthorn. C.

$\checkmark$ Cenanothus Americanus, L. Red.root. A.

Order 31.

CELASTRACEAE. STAFF-TREE FAMI,Y.

$\checkmark$ Euonymus Americanus, L. Strawherry Bush. A.

atropurpureus, Jacq. Purple Strawberry Bush. C.

Europaeus, L. Spindle Tree. C.

$r$ Celastrus scaudens, L. Bittersweet. W.

Pachystima Canbyi, Gray. Canby's Mountain Lover. W.

Order 32.

ILICACEAE. HOLLY FAMILY.

-ILEX opaca, Ait. American Holly. A.

Cassine, l. Daboon Holly. E.

rvomitoria, Ait. Yaupon Holly. E.

glabra, L. Inkberry Holly. E. and C.

decidua, Walt. Meadow Holly. E. and C.

monticola, Gray. Large-leared Holly. W.

mollis, Gray. Broad-leaved Holly. W.

laevigata, Gray. Smooth Winterberay. E.

myrtifolia, Walt. Myrtle-leaved Holly. E.

ambigua, Chapm. Serrateler ved Huilly. E.

longipes, Chapm. Smcoth-leaved Holly. E.

Amelanchier, Curtis: Amelanchier Holly. E

-verticillata, Gray. Black Alder. A.

Beadlei, Ashe. Beadle's Holly. C. and W.

lucida (Ait), T. \& G. Inkberry. E. 
Orier 33.

CYRILIACAE. CYRILLA FAMITY.

Crricha racemiflora, Walt. Leatherwood. E.

Order 34.

STAPHYLEACEAE. BLADDER-NUT FAMILY.

Staphylea trifolia, L. Bladier-uut. A.

Order $3 j$.

SAPINDACEAE. SOAP BERRY FAMILY.

Cardospernum Halicacabum, L. Balloon Vine. C. $\checkmark$ Esculus glabra, Willd. Fetid Buckere. W. octaudra, Marsh. Sweet Buckeye. W'.

$\downarrow$ Pavia, L. Red Buckeve. C. and W.

Acer saccharinum, L. Silver IIaple. IV.

rubrum, L Red Maple. A

- Saccharum. Marsh. Sugar Maple. IV.

Saccharum Floridanum. Small. Sugar Maple. C.

Pennsylranicum, L. Guasefoot Map'e. II'.

spicatum, Lam. Mountain Maple. $\mathrm{W}$.

rNegundo, L. Ash.leaved Miple. A.

Order 36.

POLYGALACEAE. MILKWORT FAMILY.

Polygata cymosa, Walt. Tall Pine barren Milkwort. ramosa, Ell. Low Pine-barren Milkwort. lutea, L. Orange Milkwort. cruciata, L. Marsh Milkwort. brevifulia, Nutt. Short-leaved Milkwort. verticillata. L. Whorled Milkwort. ambigua, Iutt. Lnose spiked Milkwort. incarnata, L. Piuk Milkwort. sanguinea, L. Purule Milkwnrt. Curtissii, 'Gray. Curtiss' Milkwort. Mariana, Mill. Maryland Milkwort. Nuttallii, T. c G. Nuttall's Milkwort. Sentga, L S-neka Suakeront. polygama, Walt. Racemed Milkwort. paucifolia, Willd. Fringet Milkwort.

Order 3i.

LEGUINIYOSAE. PLLSE FAMILY.

Crotallaria sagittalis, L. Rattlebox. E. and C. ovalis, Pursh. Prostrate Rattle-box. C. and W. Lupisus perenuis, L. Wilr Lupine. E. and C. perennis gracilis, Nutt. Slender Lupine. E. and C. 
Luprous villosus, Willd. Hoary Lupine. E. and C.

diffusus, Nutt. Prostrate Lupine. E. and C.

Medicago lupulina, L. Black Medic. E. and C.

denticulata, Willd. 'Toothed Medic. E. and C.

sativa, L. Purple Medic. E and C.

Crtissus scoparius, L. Scotch Bronm. C. and W.

Melilotes alba, Desv. White Melilot. A.

officinalis, Lam. Yellow Mellilot. A.

parviflora, D sf. Anuual Melilot. A.

Indica, Ail. Indian Melilot E.

Thifolium agrarium, L. Yellow Hop Clover. A.

procumbens, L. Smaller Hnp Clover. A.

dubium, Sibth. Least Hop Clover. A.

rincarnutum, L. Crimson Clover. A.

$\checkmark$ arrense, L. Rabbit-foot Clover. A.

pratense, L Red Clover. A.

reflexum, L. Buffaloe Clorer. A.

bybridum, L. Alsatian Clover. A.

Carolinianum, Michx. Carolina Clover. A.

repens, L. White Clover. A.

Hosackia Purshiana, Beuth. Trefoil. C.

Lotus Helleri, Britton. Heller's Lotus. C.

Psoralea melilatoides, Michx. Psoralea. C.

Onobrychis, Nutt. Psoralea. C.

Canescens, Michx. Psorilea. E.

Lupinellus, Michx. Psoralea. E.

Petalostemon corymbosus, Michx. E. corymbosus trifoliatus, Chapm. E.

Ayorpha fruticnsa, L False Indigo. A.

herbacea. Walt. Lead Plaut. E.

glabra, Desf. Lead Plant. W.

virgata, Small. Lead Plant. WW.

Robrisa Pseudacacia, L. Locust. A.

viscosa, Tent. Clammy Locust. W.

hispida, L. Inss Locust. C. and IT.

hispida rosea, Pursh Ro:e Locust. C. and IT.

Wistaria frutescens, D C. Amprican Wisteria. E. and C.

Teprosia Virginica, Pers. Goat's Rue. A.

spicata, T. E G. Hirsute Goat's Rue. C. and W.

hispidula, Pursh. Aispid Goat's Rue. E. and C.

ambigua, Cursis. Hoary Goat's Rue. E. aud"C,

Isdigofera Caroliniana, Walt. Carolina Indigo.!cE.

tinctoria, L. Indign. E

Anil, L. Indigo. E.

Sesbania vesicaria, Ell. Sesban. E.

macrocarpa, Muhl. Sesban. E.

punicea, Beuth. Seshan. E.

Astralagus Canadensis, L. Milk Vetch. W. 
Astralagus glaber, Michx. Vetch. E.

Vicia satira, L. Common V'etch. A.

birsuta, Koch. Hairy Vetch. E.

Cracea, L. Cow Vetch. E. and C.

Americana, Muhl. American Vetch. E. and C.

Caroliniana, Walt. Carolina Vuch. A.

tetrasperma, Moluch. Slenter Tetch. E. and C.

angustifolia, Roth. Smaller Cimmon Veteh. E. and C.

Lathyrus vemosus, Muhl. Trimy P.a. C.

Emyrtifolius, Mubl Myrtle.leaved Pea. W.

Aeschyosexe hispida, Willd Juinted Tetch. C.

Zorxia tetraphylla, Michx. Zoruia. E

Stylosanthes elatior, Swariz. Peicil Finwer. A.

riparia, Kearney. Prucll Flower. IV.

Lespedeza repeus, Burt. Cre ping Bush-clwer. A.

violacea, Pers. Vur et Bu-h-clover. A.

reticulala, Pers. Bu-liclucer. A

Sturei, Nutt. Sture's Bush clover. E and C.

striata, Hook \& Am. J.1 an Bus -clover. A.

hirta, Ell. Haily Buslı.clıver. E. anu C.

hirta oblongifolia, Britlon. Bush-clover. E. and C.

capitata, Michx. Ruund-headed Busl-clover. C. and W. capilata seric a. H ok \& Arus. Bush-chuer. E. procumbens, Michx Triling Bu-h chocer. E and C. Nuttallii, Ditrl. Nuttal's Bu-h clorer. E.

frutescens (L), Brittum. Wand-like Bush-clover. E. and $\mathrm{C}$,

Virginica (L.). Britıon. Sltnder Bush-clover. E. and C. angustifolia (Pur-l,.), Ell. Narrow leaved Busb-clover. E. and $\mathrm{C}$.

Desmodium nudiflurum, D C. Naked Beggar'stick. A. acuminatum, D C. Punndleavel Beggar's tick. A. pauciflorum, D). ('. Few fluwerel Beggar's tick. A. lineatum, D C. Simt B guas's-tick. C. rotundifolium. D C. Pritrite Btgrgar'stick. A. ochroleucum. Cur i-. ('ram fl wrorul Beggar's-tick. C. humifu-um, Brck. Trislong Beggirl's-lick. E. and C. strictum, D. ('. vilff L wagr's ilick. E. sessilifolium, T \& $G$ simile-leared Beguar's-tick. E.

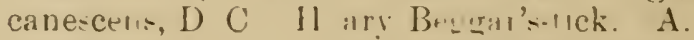

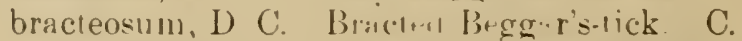
paniculatum, D C. I'a icied B. gragr': tick. E. paniculatum Clı mminıi. Liritın. C'sapman's Beggar'stick. E. paniculatum 1.uh 1, $V^{\top}$ il Pabescent Btggar'stick. E. laevigatum, D. ' s " h B. gear's tirk. A. rhombifulum. D (' li umh feaved Beggar's-tick. E. viridifl rum, B. r.k. $V$.. leaver B ggar's-tick. C. 
Desuodium Dillenii, Darl. Dillen's Beggar's-tick. A.

Canadense, D. C. Canadian Beggar's-tick. A.

rigidum, D. C. Rigid Beggar's tick. A.

Marylandicum, Bott. Smooth Beggar's-tick. E.

ciliare, D. C. Hairy Beggar's tick. E. \& C.

tortuosum, D C. A.

cuspidatum, T. \& G. A

glabellum, D. C. E.

tenuifolium, T. \& G. E.

Rhychosia tomentosa, H. \& A. Twining Rhynchosia. A. erecta, D. C. Erect Rhynchosia. A.

simplicifolia, Wood. Round-leaved Rhynchosia. E. and C.

Phaseolus perennis, Walt. Wild Bean. A.

helvolus, L. Trailing Wild Bean. A.

umbellatus, Brittun. Pink Wild Bean. E.

Apios tuberosa, Muluch. Gruund Nut. A.

Tigra luteola, Bentlı. E.

ERythrisa herbacea, L. E.

Clitoria Mariana, L. Partridge Pea. A.

Cestrosena Tirginiana, Benth. Virginia Partridge Pea. C. and W. Airphicorpaea monoica, Nuit. Hng Pea. E. and C.

Galactia glabella, Michix. Milk Pea. E. and C.

pilosa, Ell. Downy Milk Pea. E. and C.

mollis. Michx. Hoary Milk Pea. E.

sessiliflora, T. \& G. Sessile-flowered Milk Pea. E.

Baptisia lanceolata. Wild Indign. E.

villosi, Ell. Villnus Wild Indigo. E.

tinctoria, R Br. Jellow Wild Indigo. A.

alba, R. Br. White Will Indigo. W.

leucantha, T. \& $\mathrm{G}$ Laıgea Wild Indigo. E. and C.

Thermopsis Caroliniana, Cnrlis. Mountain Thermopsis. WV.

fraxinifolia, Curtis. Smaller Mountain Thermopsis. IV'.

mollis, Curtis. Hairy Thermop-is. C.

Cladrastis tinctoria, Raf. YHlliw wood. W.

$\checkmark$ Cercis Canarlensis, L. Judas T'ree. C. and WV.

Cassia nictitans, L. Sensitive Pea. A.

- Chamaecrista, L. Large fl wered Sensi ive Pea. A. nbtusifolia, L. Low.flowered Sensitive Pea. C.

Marylandica, L Wild Senna. C. and IT. occidenta is, L. Coffee Senur. IV.

aspera, Ell. Hirsule Sensitive Pea. A.

$\checkmark$ Gleditschia triacanthos, L. Honey Locust. C. and W. monosperma, Walt. Swamp Locust.

Misosa. EC.

Schraxkia uncinata, Willd. Sensitive Brier.

angustata, T. \& G. Narrow-leaved Sensitive Brier. W. 


\section{Order $3 \$$.}

POSACEAF. ROSE FAMILY.

Chrisobalaxts oblougifolius. Michx. E.

Prexus Americana, Marsh. Wild Red Plum. A. Chicasa, Michx. Hog Plum. A.

Pemnsylranjea, L. IVild Peil Chrory. W.

$\checkmark$ serotina, Ehrhart. Wild Black Cherry. W'.

$\checkmark$ Tirginiana, L. Choke Bherry. A.

Caroliniana. Air. Muck Orange. IV.

cuneata, Raf Aplalachian Cherry. W.

Arium. L. Sweet Cherri. W.

Amigdalcs Persica. L. Peach. A.

SpIPAeA opulifolia, L. Ninebark. C. and WV.

opulifolia ferruginea, Nurt. Pubescent Ninebark. C. and WV. corymbosa, Rit. Corymbed sijraea. IV.

towentosa, L. Hardiack A

salicifulia, L Willow lenvel spiraea. A.

Jobata, Murr. Lohed Sinrapa. IV.

Truncus. L. Grat s.beard Spirdea. C. and W.

Virginiana, Bruton. Tirgina Spirata. $\mathbb{H}^{\circ}$.

GillexiA trifoliata, Mn]uch. Iudiau Physic. C. and IV sipunlacta, Nuit. Anerjean I pecac. $C_{\text {- }}$ and $W$.

Agrisonia hirsuta, Bich. Tall hairy Agrimony. C. and W. striata. Mirebx. Wuodlaid Agimony. A.

pumila, Mubl. šmall-fruitel Agrimuny. E. and C. mollıs, T. \& $G$ Soft Agrimony. IV.

parviflora, Soland. Many flowered Agrimony. C. and W.

Eupatoria, L. European Agrimony. ('. and IV.

SANGuisorba Canadensis, L. Americall Burnel. iV.

ALCHEMILLA arvenis. L. Firegrass. A

Gecm Tirginianum. L. Tirginia Avens. C.

album, Gunelin. Mhice Arsis. $C$ and 16.

flavum, Purer Cream Arens. IV.

geniculatum, Mich. Hairy Arens. W.

vernum, T. \& G siring Aiens. W'.

radjatum, Mchx. Pnan Mounaain Aolus. W.

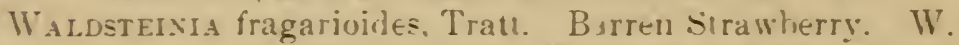
parviflora. Small. Soullieru Dry stiawberr: TV.

Potextilla Norvegic, L Prough Cinqurfoil. A.

Canadensis, L Field Cinquefoil. A.

Canarlensis pumila, "T. $G$ G Cinqueforl. A.

tridentata, Soland. Three wotherl Curquefoil. IV.

recia, L. Rough-fruitil Cinquefoil. IV.

- Fragaria Virginiana. Uuch. Virginia strawlery. A.

vesca, L. European Sirawherry. A.

Indica. Andr. Indian Strawberry. A. r Rubls odoratus, L Rusefflowering Raspberry. W. 
Rubus strigosus, Michx. Wild Red Raspberry. IV.

- occidentalis, L. Black Raspberty. W.

- villosus, Ait. High Bush Blacklerry. A.

frondosus, Bigil. Bracter Blackberry. A.

Millspaughii, Britton. Millspaugh's Blackberry. IV.

parviflnrus, Walt. Sand Blackberry E. and C.

hispidus, L. Swamp Blathlierry. WW.

trivialis Michx. Low hush Blackberry. A.

Baileyanus, Brittor. Bailey's B ackherry. C.

Canadensis, L. Dewherry. A.

Rosa setigera, Michix. Climbing R se. C.

- Carolina, L Swamp Rnse. A

rubiginosa. L. Sweethrier Rose. C.

- humilis, Marsh. Paslure Rose. A.

laerigata, Michx. Ch. r.kee Rose. A.

Crataegus Crus.Galli, L Cockspur Thorm. A.

spallulara, Michx. Small-fruited Haw. E. and C.

punctati, Jacq. Large-fruited Haw. IV.

cordata, Ait. Wishington $\mathrm{H}$ a $W$. C. and W.

roiusdifolia, Borck. Haw. $\mathbb{W}^{T}$.

Oxycantha, L May l'horn. (C

elliptica Ait. May Thurn. W.

rapiffolia, Michx. Parsley Haw. E and C.

$\checkmark$ coccinea, L Red Haw. A

glandulusa, Willd. Glandular H+w. C. aud W.

inacratcantha, Lndi. Long spine Haw. C.

mollis, Scheele. Rerffrumed Haw. C.

tomentosa, L. Prar Haw. C.

tomentosa Chapmanii, Beadle. Chapnan's Haw. W.

flava, Ait. Yellow Haw. A.

uniflora, Muluch Dwarf Haw. E and C.

Vailiae, Britton. Vail's Haw. C.

collina, Cha:m. Haw. IV.

Pyrus Americana, D. C. Mountuin Ash. WV.

- angustifolia, Ait. Narrow leaved Crab Apple. C.

coronaria, L. American Cr.⿲丿 Apple. C. and WV.

Malus, L. Common Auple. C.

arbutifolia, L f. Red Chuke-herry. C.

arbutifnlia nigra, Willd. Black Choke-berry. C. and WW.

arbutifolia erytlirucarpa, (hall. Choke-berry. C. and W.

Amelaxchikr Canadensis, Medic. June berry. A.

Botryapium, D. C. Shar-busli. A.

rotundifolia, Roem. Round-leaved Shad-bush. A.

Order 39.

CALYCANTHACEAE. ALL SPICE FAMILY.

Calycanthus floridus, L. Sweet Shrub. A.

laevigatus, Willd. Pubescrnt Sweet Shrub. C. and W. glaucus, Willd. Smooth Sweet Shrub. C. and W. 


\section{Order 40.}

SAXIFRAGACEAE. SAXIFRAGE FAMIIT.

Astrlbe decandra, Don. Goat's Beard. C. and WV. Saxirkaga Pennsylvanica, L. Pemnsylvania Saxifrage. C. micranthifolia, B S. P. Lettuce Saxifrage. IV. Virginiensis, Michx. Early Saxifrage. C. and W. Grayana, Britton. Gray's Saxifrage. W.

Michauxii, Britton. Michaux's Saxifrage. W. Careyana, Gray. Carey's Sixifrage. IV.

Heuchera Americana, L. American Heuchera. C. and W. villosa, Michx. Hairy Heuchera. C. and W.

Curtissii, Gray. Curtiso' Heuchera. IV. Rugellii, Sisuttlw. Rugel's Heuchera. W. pubescens, Pursh. Downy Heuchera. IV. hispirla, Pursh. Rough Heuchera. W.

Boykrnia aconitifolia, Nutt. Aconite Saxifrage. W.

Trarella cordifolia, L. False Nitre wort. W.

Miterla diphylla, L. True Nitre wort. W.

Chrysosplenium Americanum, Schw. Golden Saxifrage. W. rIteA Virginica, L. Virginia Willow. A LHyorangea arborescens, L. Wild Hvdrangea. C. and W. radiata, Walt Downy Hydrangea. W.

Decumaria barbara, L. Decumaria. E. and C. $\checkmark$ Philadelphus grandiflorus, Willd. Largeflowered Syranga. W. hirsulas, Nutt. Hairy Syringa. W.

- inoriorus. L. Scentless Syringa. C. and IV. reoronarius, L. Gurdon Syringa. C.

rRises Cynosbati, L. Wild Gooseberry. W. rotundifolium, Michx. Eastern IVild Gooseberry. W. prostratum. L'Her. Fetid Gonseberry. W. resinosum, Pursh. Resinous Gooseberry. W. Uva-crispa, L. Garden Gooseberry. (j. floridum, L'Her. Wild Black Currant. C.

- rubrum, I. Red Currant. C.

Order 41.

PARNASSIACEAL. PARNASSIA FAMILY.

Parnassia Caroliniana, Michx. Grass-of-Parnassus. A. asarifolia, Vent Mountain Grass of Parnassus. „. W. grandiflora, D. C. Large-leaved Grass-of-Parnassus. W. Oriler 42.

CRASSULACEAE. ORPINE FAMILY.

Sedur roseum, Scop. Rosewort. W. telephioides, Michx. American Orpine. W. pulchellum, Michx. Widow's Cross. IV. ternatum, Michx. Wild Stonecrop. W. 
Sedum Nevii, Gray. Nevius' Stonecrop. IV.

Rhodiola, D. C. Canby's Stonecrop. W.

Penthorium sedioides, L Virginia Stonecrop. A.

Bryophyllum calycinum, Salisb. Bryophyllum. C.

Diamorphia pusilla, Nutt. Diamorpha. C. and W.

Order 43.

HAMAMELACEAE. WITCH HAZEL FAMILY.

$\checkmark$ Hamamelis Virginica, L. Witch Hazel. A.

Fothergilia alnifolia, L. Dwarf Alder. E. and C. monticola, Ashe. Dwarf Alder. W.

Liquidambar Styraciflua, L. Sweet Gum. A.

Order 44.

MELASTOMACEAE. DEER GRASS FAMILY.

Rhexia Mariana, L. Maryland Meadow-Beauty. A. Virginica, L. Virginia Meadow-Beauty. A. glabella, Michx. Purple Meadow-Beauty. E.

ciliosa, Michx. Ciliate Meadow-Beauty. E. and C. lutea, Walt. Yellow Mleadow-Beauty. E.

Order 45.

LYTHRACEAE. LOOSESTRIFE FAMILY.

Didiplis linearis, Raf. Water Purslane. E.

Ammana humilis, Michx. Ammania. E.

Koehnei, Britton. Koehne's Ammania. E.

latifolia, L. Ammania. E.

Lythrum alatum, Pursh. Angled Loosestrife. A.

lineare, L Linea Loosestrife. E.

Nesaea verticillata, H. B. K. Swamp Loosestrife. E. and C.

Cuphea viscosissima, Jacq. Wax-weed. C. and W.

Orier 46.

HALORAGEAE. WATER-MILFOIL FAMILY.

Proserpivaca palustris, L. Mermaid-weed. E.

pectinacea, Lam. Cut-leaved Mermaid-weed. E.

Mrriophylum verticillatum, L. Whorled Water.Milfoil. E. spicatum, L. Spiked Water-IIilfoil. E.

heterophyllum, Michx. Various-leaved WaterMilfoil. E.

scabratum, Michx. Pinnate Water-Milfoil. E.

Order 4i.

ONAGRACEAE. EYENING PRIMROSE FAMILY.

GaURA biemnis, L. Biennial Gaura. W.

filipes, Spach. Michaux's Gaura. C.

angustifolia, Michx. Field Gaura. C. 
Oenothera biennis, L. Hairy Evening Primrose. A. humifusa, Nutt. Seaside Evening Primrose. E and C. sinuata, L. Sinuate Evening Primrnse. E. and C. glauca, Michx. Smooth Evening Primrose. WV. fruticosa, L. Common Evening Primruse. A.

- linearis, Michx. Narrow Evening Primrose. A. pumila. L. Small Erening Primruse. C. grandiflora, Ait. Grund Evening Primrnse. A. longipedicellata, small Long-stemmer Evening Prim. rose. W.

pilosella, Raf. Mountain Evening Primrose. W.

Epilobium strictum, Mulıl. Soft Willow-herb. W.

Coloratum, Muhl. Purple Willow herb. W.

angustifolium, L Tall Willow-herb. II.

palusire(L lineare, Cray. Mountain Willow-herb. W'.

Jussiaea!decurrens, D C. Primrrise-Willow. A. -3... f́diffusa, Forkl. Floating Primrose-lWillow. E.

Ludwigra palustric, Ell. Marsh Parslane. A. alternifolia, L. Alternated Ludwigia. A. virgata, Michx Teree Ludwigia. E. hirtella, Raf. Hairy Ludwigia. E. linearis, Walt. Linerleawn Ludwigia. E. linifolia. Poir. Smonth Lindwigia. E. pilosa, Walt. Tomentose Ludwigia. E. sphaerocarna, Ell. Glohe-fruitul Ludwigia. E. and C. capitata, Michx. Capitate Ludwigia. E. and C. alata, Ell. Wing-semmed Ludwigia. E microcarpa, Michx. Peralless Ludwigia. E. and C. arcuata, Walt. Curve modred Ludwigia. E

Circaea Lutetiana, L. Enchanter's Nightshade. C. and W'. alpina, L. Lesser Enchanter's Nightshade. C. and W.

Order fs.

TURAERACEAE. TURNERA FAMILY.

Pirigukta fulva, Chapm. Pirigueta. E. Oriler 49 .

PASSIFLORACEAE PASSIUN FLOWER FAMILI.

Passiflora incarnata, L Mar Pop. A. lutea, L. Small Iay Pop. A.

Order 50 .

CLCURBITACEAE. GUURD FAMILY.

Lagexaria vulgaris, Sering. Gourd. A. Melothria pendula, L. Melothria. E. Sicyos angulatus, L. One seeded Cucumber. C. 
Order 51.

CACTACEAE. CACTUS FAMILY.

-Opuntra vulgaris, Mill. Prickly Pear. A.

Order 5:?

\section{FICOIDEAE.}

Sesuviumiportulacastrum, L Sea Purslane. E. Ipentandrum, Ell. Sea Purslane. E. Mollugo,verticllata, L. Carpet-weed. A.

Order 5.5 .

UMBELLIFERAE. PARSLEY FAMILY.

Hydrocotyle umbellata, L Umbellate Water cup. E.

Canbyi C. \& R. Canby's Water cup. E.

rerticellata, Thurb. Whorled Water.cup. E.

Americana, L. American Water-cup. A.

ranulculoides, Lf. Floating Water-cup. E.

Bunariensis, Lam. E.

repanda, Pers. Ova e Water-cup. E.

Crantzia!lineata (Michx.), Nutt. Crantzia. E.

SAxicula Marylandica, L. Black Sanicle. A.

Canadensis. L. Canada Sanicle. A.

igregaria, Bicknell. Clustered Stnicle. E. and C.

Erysgivis anccaefolium, Michx. Button Snake Root. A.

yuccaefolium synchaetum, Gray. Button Snake Root. E. virgatum, Cam. Virgate Button Suake Root. A.

Virginianum, Lam. V'irginia Button Snake Ront. A.

praealtum, Gray. Marsh Button Snake Root.

Prmpersella jutegerrima, B. \& H. Yellow Pimpernel. E. aud C. Bupleuruar rotundifolium, $L$ Hare's Ear. A.

Cicuta maculata, L. Poi-on Hemlock. A

Aprum Petroselinum, L. Garden Parsley. É. and C.

graveolens, $\mathrm{L}$ Celery. $\mathrm{E}$ and $\dot{\mathrm{C}}$.

leptophyllum, D. C. Marsh Parsley. E. and C.

Leptocaulis divaricatus, D. C. E.

Discopletra capillacra. D. C. Bishop wed. E.

Deringa Canadensis, D. C. Honewort. C. and IV.

Sium lineare, Michx. Water Parsnip. A.

longifolium, Pursh. Water Parsnip. E. and C.

Thaspium trifoliatum (L.), Britton. Golden Alexander. C. and $W^{2}$.

aureum, Nutt. Golden Alexander. A.

barbinode, Nutt. Hairy Golden Alexander. A.

pinuatifidum, Gray. Cur leaved Golden Alexander. WV.

Ligusticum actaeifolium, Michx. Angelica. IV.

Zizia aurea, Koch. Golden Meadow Parsnip. A.

Bebbii, C. \& R Bebh's Meadow Parsnip. W.

cordata, D. C. Heart-leaved Ileadow Parsnip. A. 
Axgelica Curtissii, Buckley. Curtiss' Angeliro. WV. hirsuta, Muhl. Pubescent Angelico. A.

Conioselinum Canadense, T. \& G. Hemlock Parsley. IV.

Tiedrania rigida, C. \& R. Water Drop-wort. E. und C. teretifolia, D. C. Water Drop-wort. E. and C. teruata, C. \& R. Ternate IV ater Drop-wort. E. and C. Heracleum lanatum. Michx. Cow.Parsnip. IV.

Daucus Carota, L. Wild Cฯrrot. A. pusilla, Michx. Wild Carrot. A.

ChaeropuyluUm procumbens. Lam. Chervil. A.

Teinturieri, Hook. Chervil. E. and C.

Osmorrhiza brevistylis, D. C. Sweet Cicely. C. \& W. longistylis, D. C, Sweet Cicely. C. and W.

Erigeria bulbosa, Nutt. Harbinger of Spring. W.

Coriandrum sativum, L. Coriander. C. and W.

Caucalis nodosa, Huds. Herge Parsley. E.

Pastinaca sativa, L. Wild Parsnip. A.

EÆthusa Cynapium, L. Fool's Parsley. E.

Faniculum vulgare, Gaert. Fennel. C.

Scandix Pecten.Veneris, L. Shepherd's-needle. E. aad C.

Carum Carui, L. Caraway. C.

Order 54.

ARALIACEAE. GINSENG FAMILY.

vAralia racemosa, L. Spikenard. W.

hispida Mich. Rough Sarsaparilla. WV.

nudicaulis, L. Wild Strsaparilla. IV.

r spinosa. Prickly Ash. A.

Paxax quinquefolium, L. Ginseng. IV.

trifolium, L. Dwarf Ginseng. E.

Order jij.

CORNACEAE. DOGWOOD FAMILY.

- Connus florida, L. Flowering Dogwood. A. circinata, L'Her. Round-leaved Dogwood. W.

- sericea, L. Swamp Dogwood, E. and C.

- stolonifera, Michx. Red Osier Dogwood. W.

paniculata, L'Her. Panicled Dogwnod. C. and W.

stricta, Lam. Strict Dogwood. A.

- alternifolia, L. f. Alternate-leaved Dogwood. C. and W.

-Nyssa sylvatica, Marsh. Tupelo. A.

biflora, Walt. Water Tupelo. E.

- aquatica, L. Larger Water Tupelo. E.

Caroliniana, Poir. Carolina Tupelo. E.

Order 56.

CAPRIFOLIACEAE. HONEYSUCKLE FAMILY.

- Sambucus Canadensis, L. American Elder. A.

pubens, Michx. Red berried Elder. W. 
Viburnum alnifolium, Marsh. Hobble-bush. W.

- acerifolium, L. Arrow-wood. A.

pubescens, Pursh. Downy Arrow-wond. IV.

deutatum, L. Mealy-tree C. and W.

molle, Michx. Soft-leaved Arrow-wood. E.

rassinoides, L. Withe-rod. W.

nudum, L. Larger Withe-rod A.

rLentago, I. Sheep-berry. W.

prunifolium, L. Black Haw. A.

$\checkmark$ rufotomentosum, Small. Southern Black Haw. A. obovatum, Walt. Small Black Haw. E.

angustifolium, T. \& G. Swamp Withe-rod. E.

Triosteum perfoliatum, L. Fever-wort. C. and IV.

angustifolium, L. Narrow Fever-wort. C. and IV.

$\checkmark$ Symphoricarpus racemosus, Michx. Snow berry. C.

$\checkmark$ vulgaris, Michx. Coral-berry. C. and IV.

- Lonicera Caprifolium, L. Italian Honeysuckle. C. and W.

parviflora, Lam. Glaucous Honersuckle. W.

flava, Sims. Xellow Honeysuckle. IV.

sempervirens, L. Trumpet Honeysuckle. A.

Japonica, Thumb. Japanese Honeysuckle. A.

$\checkmark$ Diervilla trifida, Moluch. Bush Honeysuckle. W.

sessilifolia, Buckley. Sessile-leaved Honeysuckle.: W. rivularis, Gattinger. Mountain Honeysuckle. IV.

Order 57.

RUBIACEAE. MADDER FAMILY.

$\checkmark$ Houstonia coerulea, L. Bluets. Innocense. A.

serphyllifolia Michx. Thyme-leaved Innocense. A.

minor (Michx.), Britton. Small Innocense. E. and $₫ \mathrm{C}$. purpurea, L. Large Innocense. IV.

purpurea pubescens, Britton. Hairy Innocense. W. purpurea calycosa, Gray. Narrow-leaved Innocense. C. and IV.

longifolia, Gaertn. Long-leaved Innocense. C.

tenuifolia, Nutt. Slender Innocense. A.

Oldexlandia glomerata, Michx. Clustered Bluets. E.

Cephalanthus occidentalis, L. Button-bush. A.

Mitchella repens, L. Partridge-herry. A.

Drodia teres, Walt. Rough Button weed. A.

Virginiana, L. Larger Button-weed. A.

Galium Parisiense, L. Wall Bedstraw. C.

Aparine, C. Cleavers. A.

pilosum, Ait. Hairy Cleavers. E. and C.

puncticulosum, Michx. Glabrous Cleavers. E. and C.

lanceolatum. Torr. 'Torrey's Cleavers. W.

circaezans, Michx. Cross Cleavers. A.

triflorum, Michx. Fragrant Cleavers. A. 
Galium latifolium, Michx. Purple Cleavers. W. tinctorium, L Wild Madder. IV.

tinctorium filfolium, Wie. L-sser Wild Madder. E Claytonj. Michx. Clayton's Madder. E. parvifl rum. Raf. Shining Marlder. E. and C. Asprellum, Michx. Rough Madder. C. and W. hispidulum, Miclix. Coast Mardrer. E. trifidum, L small Mardier. E.

Order 58.

\section{LOGANIACEAE LOGANIA FAMILY.}

Spigelia Marilandica, L. Pink Ront. E. and C. $\checkmark$ Geisenium sempervirens, Ait. $f$ Yelluw Jessamine. E. and C. Mitkeola petiolata, 'T. d G. Mirtwort. E. amd C. sessilifili:ı, T. \& G Whorled Mitrewrt. E. and C. Polyprenum procembens, L. Pulypremum. A.

Order 59.

VALEHIANACEAE. VALIERAN FAMILY.

Valeriana paucifl ira, Michx. Valerian. W.

Valerianelda Locusta, Betike. Curn salad. C.

Chenopmifulia, D. C. Goone-finot Corn Salad. C. radiata, Durf. Beaked Corn Sialad. C.

Order 60.

DIPSACACEAE. TEASEI, FAMLY

Dirsacus sylvestris, Mill.r. Teasel. C'and W.

Order 61.

CICHORIACEAE. CHICORY FAMILY.

Crchorium Intybus, L. Chicory. A. divaricatum, D. C. Chicory. W.

Apogox, humilis, Ell. C.

Ínigia Tirginica, Willa. Dwarf Dandulion. A: amplexicaulis, Nust. Viruninia Dandelın. A.

Montana, Nult. Mountan Dandelion. IY.

Daudeliou, Nutt. Grit's bard. E. and C. Tragopogon porrifoliu-, L. Opster Plant. E. and C. Taraxacum Dens lennis, Dest. Damelun. A. Soxchus oleraceus, L Sow Thisile. A. asper, All. Siny Sow Thisle. A.

Lactuca Canadensis, L. Wild Letluce. A. hirsuta, Muhl. Hairy Wild Letture. W. integrifolia, Bigl. Arrow leaved Wild Lettuce. W. acumivata, Gray. Blue Wild Lettuce. C. and W. Floridana, Gaert. Florida Wild Lettuce. C. leucophaea, Gray. Tall Blue Wild Lettuce. IT. 
Lactuca leucophaea integrifolia. Gray. Blue Wild Lettuce. W. graminifolia, Michx. Wild Lettuce. E.

Pyrrhopappus Carolinanus. D. O. False Dandelion. A.

Crepis pulchra, L. Hawkshearit. C.

virens, L. Smouth Hawksheard. E.

biennis, L. Rough Hawksbeard. E

Hieraciun veliosum, L Blin dwort. A.

Marianum, Willd. Maryland Blnodwort. C.

Greenii, Porter \& Britton. Green`s Blondwort. WV.

paniculatum, L. Panicled Blondmort. W.

scabrum, Michx. Rnugh Bloolwort. W.

Gronovii. L. Gronosius' Bloodwort. A.

Nabalus altissimus. Hook. Tall White Lettuce. W.

albus, Hook. Lion's foot. A.

serpentarius, Honk. Canker-wetd. IV.

integrifolius, ('ass. Canker weed. IV.

virgatus, D. C. Slender Canker-weed. E.

crepidintus. D. C. Corymbed Canker-weed. W.

Order 69.

AMBROSIACEAE. RAGWEED FAMILY.

Iva frutescens, L. Mar:h Eliter. E -

imbricata, Walt. Seat-coat Marsh Elder. E.

Ambrosia trifida, L. Great Ragweed. C. and II'.

integrifolia, Muhl. Lesser Ragweed. C. and W.

artemisiafolia, L Roman Wurmwod. A.

Xavthicm spinosum, L. Clot-bur. A.

strumarium, L. Conkle-hur. A.

Canadense, Mill. American Cockle-bur. A.

Order 63.

COMPOSITAE. COMPOSITE FAMIY.

Vervosia Noreboracenis, Willd. Iron-weed. A.

Noreboracensis tomentosa, Malt. Pubescent Iron-meed.

A.

glauca (L), Britton. Broad-leaved Iron-weed. A.

gigantea (Wialt.). Britton. Tall Iron-weed.

oligonhylli, Michx. Naker Iron weed. E.

angustifolia, Michx. Leafy. Iron-reed. E.

Elephantopus Carmliniantu; Willd. Elephant's-foot. A.

nudatus, Gras. Smonth Elephant's-foot. A.

tomentosus, L. Woolly Elephant's-foot A.

Sclerolepis verticillata, Cars. Sclerolepsis. E.

Carphephorts tomentosus, T. \& G. E.

corrmbo-us, $T$ \& $\mathrm{G}$. E

bellidifolius, T. \& G E.

Liatris squarrosa, Hell. Blazing Star. E. and C.

intermedia, Small. Lesser Blazing Star. E. and C. 
Liatris elegans, Willd. Haudsome Blazing Star. E. and C. Helleri, Small. Heiler's Blazing Star. C. scariusu, Willil. b’utton Sillake-root. A. squarrulısi, $\|$ chx. Small Snake root. E. and C. spicata, II ills. Gial Feather. E. and C. spicata fmmlla, Lidd. Larger Feather. C. and W. graminfuia, Will\}. Fine-leaved Blazing Star. C. and W. gramuntolat pilası (Ait), Britton. Ciliate Blazing Star. E. tenuifilia, Nun. Smuo h Blazing Star. E. pauciflua, Pursh. Pubescent Blazing Star. E. gracili-, Pursh Graceful Blazing Star. E. odorarissima, Michx. Carolma Vanilla. E. panicula'a, Michx. Hairy Vanilla. E. regimontis Small. Vanila C.

Kunvia enpatorionles, L. False Boneset. A. Eupatokium purpueum, L. Quen of-the-Mleadow. A. puringtum falcatum (Michx), Britton. Narrow queenof the- M+ artow. A.

furpureum amomum, Gray. Purple Boneset. A. foeniculaceum, Wild. Hug-reed Boneset. C. s rotinum, Michx. Late fluwering Boneset. A. leucilepis, 'T. \& G. White bracted Boneset. E. alıum, L Write Boneset. C. and W. hyssupifolium, L. Hyssop-leaved Boneset. A. Torreymum, Short. 'Torrev's Boneset. C. and W. semiserratum, L C. Small-flowered Boneset. E. alti-simum. L. L Tall Boneset. IV. sessilifilım, L Bastard Boneset. W. verbennelolinm, Michx. Vervain Boneset. C. and W. rotunditol um, L. Wild Huartound. C. and W. pubrectl: Muhl. Hairy Boneset. E. and C. - perfolintum, L. Common Boneset. A truncatum, Mulsl. Traucole Boneset. A. ageratondes, L.f. White Snake-root. C. and W. aromaticum, L. Smaller Snake-root. C. and W'. crelestinum, L. Míst.flower. E. and C. cormopifoliun, Wilıd. E. pimmalifilum, Ell. E iucamitum, Walt. E.

Mrkania scandem-, Wi d. Climbing Boneset. A. Semicocalipus (minzui es. Nees. White Aster. E. and C. soliilaginens, Nees. Rough White Aster. E. and C. Iortifulius, Nees. Toothed White Aster. E. and C. Aster tenrbrusus, Burges. long-leaved Wond Aster. W. divaricatos, L. White Wood Aster. W. divalle, 11-cymulosus, Burgess. WV. divaricuns curnfolius, Burgess. W. divarlcalus deltoideus, Burgess. W. 
Aster divaricatus persaliens, Burgess. WV. divaricatus fontinalis, Burgess. IV. Claytoni, Burgess. Clayton's Aster. WV. curvescens, Burgess. Dome-topped Aster. C. curvescens umbelliformis, Burgess. C. curvescens oviformis, Burgess. C.

Schreberi, Nees Schreber's Aster. C. macrophyllus, L. Large-leaved Aster. W. Shortii, Hook. Short's Aster. W. azureus, Lindl. Sky-blue Aster. E. and C. cordifolius, L. Common Blue Aster. E. and C. cordifolius polycephalus, Purter. E and C. cordifolius alveanus, Burgess. W. cordifolius pedicellatu=, Burgess. W. Lowrieanus, Porter. Lowrie's Aster, E and C. ptarmicoides Georgianas, Small. Aster. C.

undulatus, L. Wary-leaf Aster. A. undulatus abruptifolius, Burgess A. undulatus loriformis, Burgess. A. undulatus triangularis, Burgess. A. patens, Ait. Late Purple Aster. A. phlogifolius, Muhl. Thin-leaver Purple Aster. A. Novae Angliae. L. New Engl nd Aster. E. and C. oblongifolius, Nutt. Aromatic Aster. E. and C. puniceus, L. Rer-stalked Aster. A. puniceus firmus, Nees. Rer-stalked Aster. A. laevis, L. Smooth Aster. E. and C. conciuus, Willd. Narrow-leaved Smooth Aster. E. purpuratus, Nees. Southern Smooth Aster. E. Novi-Belgii elodes, T. \& G. Aster. E.

Novi-Belgii Atlanticu=, Burgess. Atlantic Aster. E. Novi Belgii Brittonii, Burgess. Britton's Aster. E. concolor, L Easteru Silvery Aster. E. grandiflorus, L. Large flnwered Aster. E. and C. surculosus, Michx. Creeping Aster. E. and C. gracilis, Nutt. Tuber Aster. A. acuminatus, Mich. Mountain Aster IV. dumosus, L. Bushy Aster. E. and C. cordifolius, T. \& G. Stouter Bushy Aster. E. paniculatus, Lam. Panicled Aster. C. bellidiflorus, Willd. Aster. C. and W. simplex, Willd. Aster. C. and W. Tradescanti, L. Tradescant's Aster. C. Faxoni, Porter. Faxon's Aster. W. ericoides, L Frost weed Aster. E. and C. ericoides pilosus, Porter. Aster. W. ericoides platyphyllus, T. \& G. Aster. W. lateriflorus (L.), Britton. Calico Aster. E. 
Aster lateriflorus glomerellus, Burgess. Aster. E. lateriflorus pendulus, Burgess. Aster. E. lateriflorus horizontalis, Burgess. Aster. E. vimineus, Lam. Small White Aster. C. foliolosus, Ait. Aster. C. multiflorus, L. White Wreath Aster. A. tenuifolius L. Perennial Aster. E. subulatus, Michx. Annual Aster. E. spectabilis, Ait. Seaside Purple Aster. E. C'urtissii, 'T. \& G. Curtiss' Aster. W'. squarrosua, Walt. Aster. E.

Elliotti, T. \& G. Elliott's Aster. E. and C. infirmus, Michx. Cornel-leaved Aster. W. umbellatus, Willd. Tall Flat-top Aster. C. anygdalinus, T. \& G. Broad Flat top Aster. C. linarifolius, L Savory-leared Aster. E aud C.

Erigerox strigosus. Muhl. Daisy Fleabane. A.

an-uus. Pers. Sweet Scabious. A.

Philadelphicus, Mubl. Philadelphia Fleabaue. A. bellidifolius. Mulnl. Plantain Fleabane. E. and C. nulicaulis, Michx. Early Fleabane. E. and C. Canadensis, L. Canada Fleabone. A. linifolius, Willd. Panicled Fleabane. A.

Boltovia diffusa, Ell. Pauicled Boltonia. E. glastifulia, L'Her. Boltunia. E.

astervides, L'Her. Aster-like Boltonia. A. Bellis perrenis, L. European Daisy. E.

Solidago squarrosa, Mubl. S'out Ragged Gulden rod. A. petiolaris, dil. Downy Ragged Golden-rod. W. raesia, L Blue-stemmed Golden-rod. E. and C. flexicaulis, L. Zig-zag Golden-rod. E. and C. Curtissii, T. \& G. Curtis' Golden rod. W. bicolor, L White Golden-rod. IV. uliginosa, Nutt. Gulden rod. IV. hispida, Muhl. Hairy Golden-rud. WV. erecta, Purslı. Slender Golden-rud A. mouticola, 'T. \& G. Mountain Golden.rod. W. putuerula, Nutt. Downy Gulden-rod. E. stricta, Air. Wand-like Golden-rorl. E. specio-a, Nutt. Noble Golden-rod. A. Purshii, Porter. River-bank Ginden-rod. E. sempervirens, L Sea-side Gulden-rod. E. odura, Ait. Sreet Golden-rod. A. odora inodora, Gray. Scentless Golden-rod. A. tortifolia, Ell. Twisted-leaf Golden-rod. E and C. rugosa, Mill. Wrinkle-leaved Golden-rod. A. fistulosa, Mill. Piue barren Golden-rod. E. patula, Muhl. Spreading Golden-rod. C. and W. 
Solidago patila strictula, T. \& G. Strict Spreading Golden-rod. C. and IV:

ulmifinlia, Mubl. Elm leared Golden.rod. C. and W.

Bootiii, Houk. Brot'- Goldentrod. C. and W.

Boottii Yadkinensia, Briltm. Yadkin Golden-rod. - C.

Ellintii, T. \& G Ellint': Golden-rod. E.

juncea, Ait sharp trotherd Golden rud. A.

arguta, Ait Cint leaved Goldentrod. WV.

serutina, Ait. Late Gol.entrud. W'

glganila, Ait. Hispid Late G.lden.rod. II.

Canadensis, L. Canada Grilden-rord. C.

Canadensis seabruscula, Porter. Gulderr.rod. C.

nemoralis, Ait. Field Golden rod. C.

- rigida, L Hard leaved Goliten rod. A.

graminifolia, Ell. Fragrant Gulden-rod. C

tenuifolia, Pursh. Slender Fragrant Golden-rod. C. sphacelata, Raf. False Golden.rod. IV'.

pubens, L'urtis Pubescent Goldien rud W.

Buckley', 'T. \& G. Buckley's Gulden rod. WT.

lanciolia, T. \& G. Mnuntain Grolden-101. W.

pulverulenta, Ruit. Pine-barren Crolden-rod. E.

verna, Curtis. Hoary Pine-barren Golden rod. E. glomerata, Michx. High Wl untain Golden rod. W. spihamata, ('urtis. Tufted stemmed Golden-rod. W. angustifulia, Ell. Sea coast Golden-rod. E.

amplexicaulis. T. \& G. Clarping Golden-rod. C. plumosia, small. C:

Bigelovia nudata, D. (`. Ravles Golden-rod. E. Chrysopsis graminifolia, Nutt. Gulden deter. E.

Mariana, Nutr. Naryland Golden Aster. A.

irichophylla, Nult. Btennial Golden Aster. E. hyssopifolia, Nutt. Hissop-like Golden Aster. E. gussypina, Nutt. Cottony Golden Aster. E.

Baccharis halemifolia, L. Gromosei-bush. E glomerulifolia. Pers. Grounisel bush. E. angustifulia, Michx. (iroundsel bush. E.

Isula Helenium, L. Elecampane. IV.

Pluchea bifrons, D C. Trscid Marsh Fleabane. E. and C. foetida, D. C. Luland Marsh Fleabane. E. aud C.

camphorata, I). C. Spicy Marth Fleabane. E. and C.

Pterocatios penostachyum, Ell P'terncaulon. E.

ANTENARIA margaritacea, R Br. Everlasting. A.

plantaginifolia, Hook. Plantain Everlasting. A.

ner dioica, Greene. C'al's foot Everlastlug. C.

neglecta, Greene. Field Cat's-1n t Everlasting. C.

Graphaliugr polycephalum, Michx. Siweet Life Everlasting. A. Hell-ri, Britton. Helleı's Life Everlasting. E. and C. purpureum, L. Purple Life Everlasting. 1 . 


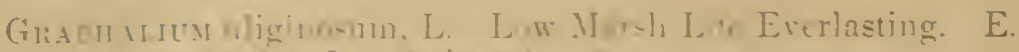

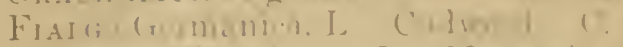

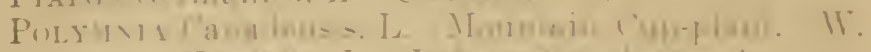

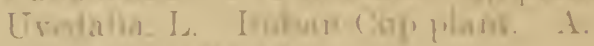

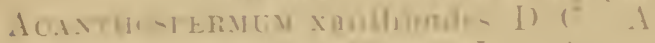

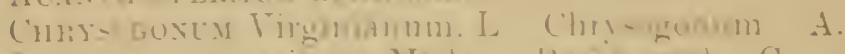

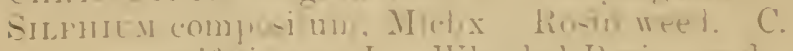

Ltrifoliatum. L. Whorlat Rosinweet. A.

Aslericu- L. Surry Risin weed. E.

Astericus dentatum. Rosin weed. E

perfoliatum, L. Indian-cup. A.

Berlanikra tomentosa, Nutt. Berlandiera. E.

Palith wium integrifolium, L. Cutting Almond. A.

auriculatum, Britton. A uricled Cutting Almoud. W.

Eclupta alba, Hassk. Eclipta. E. and C.

Borrichia frutescens, D. C. Sea Ox.ere. E.

Zixisa pauciflora, L. Zinnia. E.

$\checkmark$ Helopsis laevis, Pers. False Sun-flower. C.

gracilis, Nutt. Slender False Sun flower. C.

Tetragonotheca helianthoides. L Tetragomotheca. A.

Spilawhes repens, Michx. Spilanthes. C.

Echisacea purpurea, Mnench. Purple Cone flower. C.

- Redpeckia triloba. L. Thin-leaverl Cone-fonter. E. and C.

- hirta, L Black Eved Susan. A

Brittonii, small. Bitton's (intr.tlower. $\mathbb{W}$.

fulgidi, Ait. (range Cone-fl wer W.

spathulatia, Michx. Flathated Cone flower. C.

- lacinialia. L Tlimble weti. A.

laciniata humil- litas. Thimbleweed. A.

specicst, Neul. (inteflowtr. C'

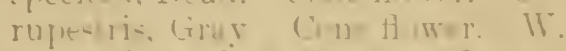

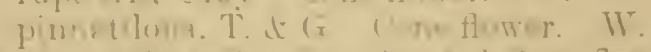

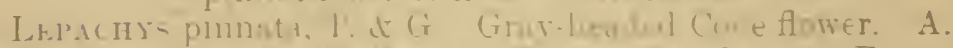

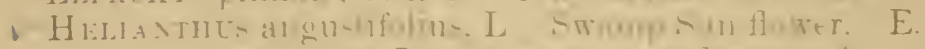

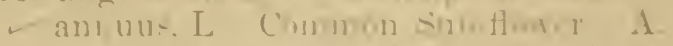

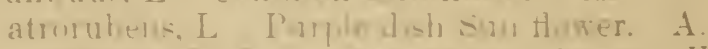

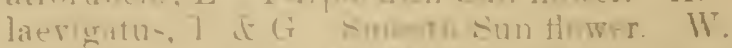

parvilimus. Benth. Small Wimd sanflimer. C. and W.

gigantens, L (iant suoflowar. II.

divaricatu-. L. Counh san-thower. IV.

strumisus. I. Pale Woolsun floxer. II.

hirsutus, Rit. stuf-mated siu flower. W'

glaucu- smull. Glumens sun-fluwer. C.

tomentuu- Michx Unally sinfluer. A.

tuberons, L. Jerusalem Articheke. 1.

heterophyllus Nult. sun.flower. E.

occidentalis (Ridd.) Dowellianus, T. \& G. Sun-flower.

II. 
Hexianturs decapetalu-, L Sunffiwer. W. Seliweinitzi, T. a $\mathrm{G}$. sim flower. $\mathbb{W}$.

Terefersat Tirgmaca. L Jirginia Crombeard. A.

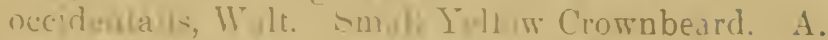

Actisoveris equarmi, Nat. Actinmeria. A.

Coneopsis lisendei, T it Swamu Tickseed. rosea, Nutt Small Ruse Tretsisel. E. senif lia. Mirhx. Greater Tick-eed. A. senif, in stellata, T. (t G. Sellate Tickseed. A. delphinifulia, Lam. Lurkspur T'rckseed, C. longif lia, Small. Tickserd. E and C. verticillata, L. Whorled Tickseed. A.

Tauceolata, L. Lance-leaved Tickseed. E. pubescens, Ell. Star Tickseed. C. and IV. crassifolia, Ait. Hairy Tickseed. C. auriculata, L Running Tickseed. A. tripteris, L. Tall Tickseed. A. aurea, Ait. Showy Tickseed. E. trichosperma, Michx. Smooth Tickseed. E. and C. latifulia, Miclix. Mountain Tickseed. WT. gladiata, Walt. Terete Tickseed. E. angustifolia, Ait. Branching Tickseed. E.

Bidess frondosa, L. Beggar's-ticks. A.

cernua, L. Smaller Beggar's-ticks. A. chrysanthemoides, Michx. Brook Beggar's-ticks. A. bipinuata, L. Spanish-neerlle Beggar's-ticks. A. connata, Muh]. Purple-temmer Beggar'stick. A. discordea, 'T. \& G. S'wamp Beggar's-ticks. E. and C. Batomrata uniflira, Ell. Baldwiola. E Garissoga parriflora, C'r Galin=oga. A. parviflora hi-pirla, D. C. Galiusoga. A. Marshaldia latifulia, l'ursh. Marshallia. A.

lanceolati, Pursh. Lonce leared Marshallia. A. lnncelata plutypylla, Curtic. Mar-liallia. 1. lacinarioides, simall. Marsballia. E. angustitolia, Pursh. Marshallia. E

$\checkmark$ Ifeceriur autumale, L. sneezawetd. A.

nudiflorum, Nutt. Purple liead Sneezereed. A. tenuifolium, Nutt Fine laved Sneezeweed. A. quadridentatum, L abill. sntezureed. W. vernale, Irall. sneezeweed. E

Curtis-ii, (rav. C'urli-' Sneezeweed. E and C'. Achillea mill-folium, $L$ Yarruw. A.

Axtremis Cotula, I. Maץ-weel. A.

arrensis, L. Field C'amomile. E. and ('. nobilis, L. (rarden C'amomile. E. and ('. tinctoria, L. Ox-eje ('amomile. E. and C. $\checkmark$ Chrisaxthemur Leucanthemum, L. Ox-eye Daisy. A. Parthenium, Pers. Feveriew. C. 
Thicetum vulgare, L. Tausy. A. vulgare crispum, D. C. Tausy. A.

Artemisia caudata, Mich. Wild Wormwood. E. Abrotanum, L. Southernwood. C. rulgaris, L. Mugwurt. C.

Arrica nudicaulis, Nutt. Leopard's bane. E. and C. Erechtites hieracifolia, Raf. Fire-weed. A.

Cacalia reuiformis, Muhl. Indian Plantain. W. atriplicifolia, L. Pale Indian Plantain. A. suaveolens, L. Wild Carawav. A.

SExecio tomentosus, Michx. Wooly Ragweed. A. obovatus, Muhl. Round-leaf Ragweed. E. and C. Balsamitae, Muhl Balsam Ragweed. IV. Smallii, Britton. Small's Ragreed. IT. Robbinsii, Oakes. Robbins' Ragweed. IT. aureus, L. Life-root. A. gracilis, Pursh. Slender Life-root. E. lobatus, Pers. Butter-weed. A. vulgaris, L. Groundsel. C. viscosus, L. Fetid Groundsel. E. Millefolium, T. \& G. Mountain Groundsel. W.

Arcticy Lappa, L. Burdock. A. minus, Schk. Common Burdock. IV.

CARDucs lanceolatus, L. Spear Thistle. A. altissimus, L.' Roadside Thistle. A. discolor, Nutt. Field Thistle. A. Virginianus, L Tirginia Thistle. A. horridulus, Pursh. Yellow Thistle. E. muticus, Pers. Swamp Thistle. E. arvensis, Robs. Canada Thistle. E. and C: repandus, Ell. Pine-barren Thistle. E.

$\checkmark$ Centaurea Cyanus, L. Corn-fiower. A. Calcitrapa, L. Star Thistle. E. Crures benedictus, L. Blessed Thislle. E. Chaptalia tomentosa, Vent. Chaptalia. E.

Order 64.

LOBELIACEAE. LOBELIA FAMILY.

Lubelia cardinalis, L. Cardinal Floṇer. A. syphilitica, L. Great Blue Lobelia. C. and IV. puberula, Michx. Downy Labelia. E. and C. leptostachys, A. D. C. Spiked Lobelia. IV. amoena, Michx. Southern Lnbelia. E. and C. amoena glandulifera. Gray Lotelia. E. and E. glandulosa, Walt. Glandular Lobelia. A. inflata, L. Tobacco Lobelia. C. and W. spicata, Lam. Pale Spiked Lobelia. C. and IV. Nuttallii, R. \& S. Nuttall's Lobelia. E. and C. 
Lobelia paludosa. Nutt. Swamp Lobelia. E. Canbyi, Gray. Caubr's Lohelia. E aud C. Cliffortiana, Gray. Clifford's Lobelia. E.

Order 6.j.

CAMPANLIACEAE. CAMPANLIAA FAMILY.

Campantla aparinoides, Pursh. Bell flower. II. diraricata, Jichx. Panicled Bell flower. W. Americana, L Tall Bell flower. II.

Spectlaria perfoliata, D. C. Large Tenus Lnoking-glass. A. biflora, Gray. Small Venus' Looking-glass. A. Order 66.

CLETHRACEAE. WHITE ALDER FAMILI.

Ciethra alnifolia, L. Sweet Pepper bush. C. and W. acuminata, Michx. Mountain Pepper bush. W.

Order bi.

PYROLACEAE. WISTERLREEN FAMILY.

Prrola rotundifolia. I. Canker Lettuce. W.

Cinmaphrica maculata, Pursh. Wintergreen. A.

umbellata, Nutt. Fragrant Wintergreen. W.

Order is.

MONOTROPACEAE. INDIAT PIPE FAMIIT.

Mosotropsis ordorata. Ell. Sreet Pine-sap. E.

L Morotropa uniflora, L. Indian Pipe. A.

Hypopitys, L. False Beech-drops. A.

Order 69.

ERICACEAE. HEATH FAMILY.

$\checkmark$ Azalea nudiflora, L. Pink Azalea. A.

canescens, Michx. Mountaiu Azalea. W.

ealendulacea, Michx Flame Azalea. C. aud $\mathrm{W}^{*}$.

arborescens, Pursh. Fragrant Azalea. C. and W.

$\checkmark$ viscosa, L. White Azalea. C. and IV.

glauca, Michx. Glaucous. White Azalea. W.

nitida, (Pursh) Britton. Shrubby. White Azalea. W.

Phodonexdrox maximum, L: Great Laurel. "W.

Catambiense, Michx. Catarba Laurel. WV.

Vaseyi, Gray. Tasey's Laurel. W. punciatum, Andr. Rose Laurel. IT.

Mexzersia globularis, Salisb. Menzeisia. W.

LeIophylia buxifolium, Ell. Sand Mrrtle. E. $\mathbb{W}$ W.

prostrtum. WV.

Kalmit latifolia, L. Mountain Laurel. C. and IV.

angustifolia. Sheep Lqurel. E. and C. 
Kalma cuneata. Michx. Swamp Luel. L: hirsuta. Walt. Hairy Liurel. E.

- Levcothue axillaris. Dun. Downy Lucuthoe. E.

$\checkmark$ Catesbaei. Gray. Catezuy Leucothe. C. and IV. recurva, (iray. Mountain Lemouthe. 11 .

- racemosa, Ciray. Swamp Lencothue. A.

Andiomena floribunda, Pursh. Feller-bush. IV. nitida, Bartr. (')mmun Fetter-bush.

- Mariaua, L Stagger bush. E. and C. vligustrina, Mubl. False Privet. C. and W. pubescer's. Small Privet. W.

$\checkmark$ speciosa, Michx. Pine-harreu Privett. E. pulverentula, (Michx.) Asíe. F. and C.

CAssand calyculata. Don. Cassaudra. II.

Oxydingum alboreum, D. C. Sour. Woud. A.

Epigalia repens, L. Trailing Arbulu $=A$.

- Galltieria procumbens. L. Checker-berry. II.

Order iu.

VACCIXIACEAE. HLCKLE PERKI FAMILY.

'Galitrsacia frondosa, T. and G. Tangle bery E and C'.

Ldumoza, T. and G. Bush Tangle terry. E. and C. hirtella, (ir y. Hairs Bu -h Tingle-berry. E. resimsa, T. and $G$. Blatk Taligle berry. E. and $C$. ursina, Gray. Talles black Tal gle verry. W. brachycera, Gray. (ilat, ous Black Taugle-berry. IV.

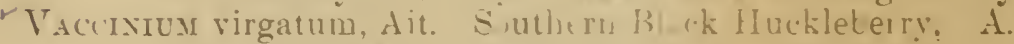
tenellum, Ait. Le-rer Black Huckleberry. E.

- corymbusum L. High bush Huckleberry. 1. fu-catum. Gray Huileberry. E.

pallidum, Ait. Mountuin Huckleberry. 11. vacillans, Kalm. I, Blue Huckleberry. W. stamineum, L. Deerberry. 1 .

arboreum, Marsh. Farkleberry. E.

crassifolium, Andr. Eastern Black Huckleherry. E. myrsinites, Michs. Hucklebery. E hirsulum, Buckles: Hairy IIuckleinergy. WV.

Chicgeres hispidula, T. and G Slowher! II.

Oxycoces macrocarpu=, Pers. Americin Crauberry. E. and $\mathbb{W}$. erythrocarpus, Pers. Mountam Cramberry. IV.

\section{Order il.}

DIAPEXSIACEAE. DIAPFXSIA FAMILY.

Pixidatuera harbulata, Michx. Fluweri g Pyxie. E. GAtax aphylla, L. Galax. C. and W.

Shortra galacifolia, Gray. "Hỵams' Eparkling Shortia." IV. 
()riler :-2.

STYRACAEAE TORAX FAMLY.

- Strata pulvemlenta, Michx. Dowiy sterax. E.

grandithora, Ait. Large leaved silı rax. A.

$\checkmark$ Americana, Lim. sumooth siturax. A.

Halesia diptera, L. sinumbep l ree. II

-tetraptera, L. snowrirn Tree, C. and IV.

Orcler is.

SYMPLOCACEAE. SWEET-LEAF FAMLY.

Srmplocus tinctoria, L'Her. Hurse-suginr. ('. and II.

Oriler $i t$.

EBENACEAE. EDUNY FAMILY.

- Diosprosos Virginiana, L. Persimmon. A.

Order $i \bar{j}$.

SAPOTACEAE SAPOULLA FAMILY.

Bunela lycioides, Peis. Buckthorn. C.

Order iti.

PRIMCIACEAE. PRIMROSE FAMLY.

Hottonia inflat., Ell. Featherficil. E.

Sanolus floribu. dus, H. B. K. Bror kwerl. E.

Lrsmachia qualdrifolia, L. Whortenl Lomeserife. C. and II.

stricta, dit. Bull, hearing Lonsestrife E.

Frastri, Duby. Fraser's Lonsestrife. IV.

asperulat folıa, Poir. Lonststrife.

Steironema ciliatum, Raf. Fringed Luosestrife. A.

tonsum, Bicknell. Southern Lor sestrife. W.

radicans, Gray. Trailing Lonsestrife. C.

lancerlatum, Grax. Lance leaved Loosestrife. E. and.C.

Axagallis arvensis, L. Red I'imperuel W.

Dodecatheor Meadia, L. American Cowship. E. and C.

Cestunculus minimu:, L. Chatfi-wetd. E.

Order is.

PLENBAGINACEAE. LFADWORT FAMILY.

Statice (aroliniana, Itale Marsh Rosemary. E.

Brasiliensis, Boiszier. Rosemary. E.

order is.

LENTIBULA ('EAL, BLADEERWORT FA.HLY.

Utricularia cornuto, Micha. Hormed Badderwort. E. and C. juncea, Tahl. Ru*h Blalderwort. E. and C. resupinata, Greene. Reversed Bladderwort. E. and.C. 
[rricularia subulata, L. '/iz zag B'adderwort. E. aud C. inflata, Walt. Swollen Bladderwort. E and (' purpurea, Walı. Purnle Blatderwort. E and C. vulgaris, $L$. Greater Bladderwort. E. aud C. tibrosa. Walt. Fibrous Bladderwort. E. gibba, L. Humped Bladderwort. E. and C:

Praficuid lutea, Walt Butterwort. E. elatior, Michx. Purple Butterwort. E. and C.

Order i9.

BIGNONIACEAL. BIGNONIA FAMILY.

Bignonia capreolata, L. Cross vine. A.

Tecoma radicans, Juss. 'Trumpet flower. A.

Catalra lignonioides, Walt. Catawba Tree. C. cordifolia, Moench. Catawha Tree. IV.

Order $\mathrm{s} 0$.

MARTYNICACEAE. UNICURN-PLANT FAMII.

Martria Louisiana, Will. Unicurn-Plant. A Order 81 .

(IROHANCHAC'EAE. BROOM RAPE FAMIIY'.

Eprphegus Virginiana, Bart. Beech-drops. A. Conophomis Americana, Wallr. Equaw-root A Aphyslox uniflorum, 'T. \& G Cancer root. A.

Order 82.

SCROPHLIARIACEAE. FIGWORT FAMIIS.

Verbacicm Thapsus, I. Great Mullein. A.

Lychuitis, L. White Mullein. A.

Blattaria, L. Moth Mullein. A.

Lrxaria Cymbalari. Mill. Coliseum Ivy. E spuria, Mill. Round leaved Toad-Flax. E.

Elatina, Mill. Cancerwort Toad-Flax. E. vulgaris, Mill. Yellow Toad.Flax. A.

Canadencis, Dumont. B ue Toarl-Flax. A. repens, Mill. Pale Blue Toar.Flax. E.

AxtirRhisum majus, L Lion's moutl, E.

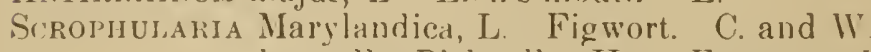
leporella, Bicknell. II are Figwurt. II.

Chelone glabra, L. Snake liear. A.

obliqua. L. Red Turtle-head. C.

Lyoni, Pursh. Lyon's Turtle head. W.

Pexstenos pubescans, Soland. Hairy Beard-tongue. (' and $W^{\circ}$. canescens, Britton. Gray Beard-tongue. C. and IT'. Digitalis, Nutt. Foxglove Beard-tongue. C: laevigatus, Soland. Smooth Beard-tongue. C. and W. 
2 Pallowisa imperialis, Sieh. Paulownia.

Mnucus ringens, L. Monkey-flower. A.

alatus, Soland. Winged Monkey-flower. E. and C.

Herpestris Munniera, H. B. K. Hedge-Hyssop. E.

nigrescens, Banth. Purnle Hedge-Hyssop. C.

amplexicaulis, Pursh. Blue Hedge. Hyssop. E.

Gratiola Vírginiana, L. Clammy Hedge-Hyssup. C. and IV. spbaencarpa, Ell. Roind-fruired Hedge Hyssop. A.

aurea, Muhl. Golden Hedge. Hyssup. E.

viscosa, Schwein. Viscid Hedge-Hyssop. IV.

Drummondi, Benth. Drummonrl's Hedge-Hyssop. IT.

officinalis, L. Hedge-Hyssop. C.

pilosa, Michx. Hairy Hedge Hyssop. E

Itrisanthes gratioloides, Benth. False Pimpermel. E.

attenuata (Muhl), Small. False Pimpornel. W.

saxicola, Chamm. False Pmpermel. IV.

Micraxthemey Nutallii, Gray. Nuttall's Nicranthemum. E. orbiculatum, Michx. Nicranthemum. E.

VEroxica Anagallis, L Speedwell. C. and W'.

Anagallis ayuatica, L. Water Speedwell. E. officinalis, L Common Speedwell. C. and W. serpyllifolia, L. Thyme leaver Spreedwell. WT.

peregrina, L. Puislane Sperdwell. W'.

arvensis, L. Curn Spredwell. A.

agrestis, L Garden Speedwell. C.

LePtaxina Tirginica, Nutt. Culver's root. C. and Wr.

Digitalis purpurea. L. Purple Fox.glove. C.

Buchnera Americana, L. Blue-hearts. C.

Sermeria tenuifolia, Purslı. Mullein Fox-glove. C. and IV. macrophylla, Nutt. Mullein Fox.glnte. $\mathbb{H}^{\text {. }}$.

Otophyla Michauxii, Bentl. Michaux's Fox-glove. E.

Dasytoma Pedicularia, Benth. Lousewort Fox glove. A.

flava, Wood. Durny Fox-glove. A.

laevigata, Raf. Entire leaved Fox glore. A.

quercifolia. Benth. Smocth Fox glove. E. and C.

pectinata, Benth. Tillions Fox-glove. E.

Gerardia linifolia, Nutt. Flax leaved Gerardia. E purpurea, L Large Purple Gerarlia. A. jurpura fasciculata, Chapm. Purple Gerardia. E. maritima, Raf. Sea-side Gerardia E tenuifolia, Tahl. Sl+nder Crerardia. A renuifolia filiformis, Chapm. Filiform Cierardia. A. Skinneriana, Wond. Skinner's Gerardia. E. auriculata, Michx. Auricled Gerardia. E and ('. aphỵlir, Nutt. Pine-barren Gerardia. E. setacea, Wait. Setaceous. Gerardia. C. Castillija coccinea, Spreng. Painted-cup. C. and II. Schwalmea Americana, L. Chaff-seed. E. 


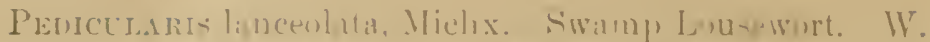
('andenats, L Betony Lotsemont A.

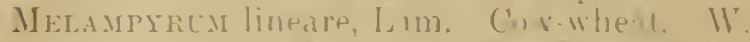
latiolium, Muhl. CuN-Wlit+1\%. II.

Oriler s:3.

SUIANACEAL FIGHTAIIALE FAMILI.

Arropa physalodec, L. Appleof Peru. Cand W.

Physals pubezens, L. Low Hairy Ground Cherry. A. Barbaclensi:, Jacq. Barbaloes (irnund Cherry. A. ohscura, Michx. Glabrou= Ciromel Cherry. A. angulata, I. C'ut-leaved Ground C'berry. C. Philadelphica, Lam. Philadelphia (round Cherry. C. lanceolata, Michx. Proirie Grumul Cherry. IV. Virginiana, Mill. Virginia Ground Cherry. A. heterophsllis, Nes. Clammy Ground Cherry. C. beteropliylla nyctagina, Rydberg. Clammy Ground cherry. C.

viccosa, L. Stellate Cromud Cherry. E.

Soldxum nigrum, l. Blakk Nightshade. A

Carolintroe. L Horse Netlle. A.

Dulcamara, L. Woodv Nightsharle. WV.

Lycopersicum, L Love Apmle. C'. aculeatisinum, Jacr. Nightshade. C.

- Lxcium valgare, Dunal Bux-lumb. (.

Carolinianum, Michx. E

Datura Siramunimm, L. Jamestown-weel. A.

Tatula, L. Purple Jamestown-weed. A.

Metel, L. Entire-leaved Jamestown-weed. E

v Nicotiana rustica, L. IVild Tuballeo. C.

$$
\text { Oriler 84. }
$$

CONVOLULACEAL. MORNIXG GLORI FAMIIY.

Dichondra repens, Forst. Dichondra. E.

Breweria humistrata. (Walt) (ray. Brewria. E. aquntica, (Walt) (iray. Water Breweria. E.

Pick+ringii, (Curlis) Gray. P'ickering's Breweria. E.

IPrnoka Quamoclit, L. American Rerl Bell-flower. E. an / C. coccinea, L. Small Red Morning glorv. A. pandurata, (1.) Neyer Wild Potan Vine. A. lacumsa, L. Small Whice Morningrglory. A. Carolina, (L) Pursls. Small Piuk Murning glory. E. purpurea, (L) Roth. Purple Morning-glory. A hederacea, Jacq. Iver-leaved Morning glory. A. sagittala, (av. Sagitate Morning glory. E. tamuifolia, Griseb. Bracted Murning glory. E. 
Cosrulrugls spithamarus I. Erright Bindweed. W. arrensis L. sivall Bimbretl A.

$$
\text { Or ler }>. j \text {. }
$$

\section{CECCLTACEAE IODWLI FHMLY.}

Cececta arvensis, Berrich. Field Dowder. A. indecora, ('hui-y. Prett! 1) odider. 1. Coryli, Engelm. H zel Dadder. W. Gronorii, Willd. L ve vise Dodder. A. rostrata, Shutaw. Beaked Dodiler. IV. compacta, Juss. ('ompract I) idder C. inflexa, Engelm. Unuel Dodder. E. and C.

$$
\text { Order } \$ 5 \text {. }
$$

POLEMONIACEAE. PHLOX FAMILY.

Phlox paniculata. L. (iarden Phlox. A. maculata, I. Wild Sweet William. C. and W. ovata, L. Mountain Phlox IV. glaberrima, I smonth Phlox. IV. glaberrima suffrutier sa. (rial. Sm.noth scabrous Phlox. II. reptans, Michx. Crawlug Phlox E. divaricata, L. Wild Blue Phlox A. amena, Sims. Hairy Phlox. C. pilosa, L. Down Phlux. C.

r subulata, L. Mos: Pink Pislox. E

Drummon tii. Houk. Drummond's Phlos. A. Polemoxitur reptans, L. Greth Vialerian. II.

\section{Order s:}

1.ENTIAXACEAE. IENTIAN FAMILI.

SABbatia lanceolata, Mait) T. and G. Lonceleaved Sabbatia. E. paniculata, (Michx Pursh. Branching Salıbatia. E. angustifolia,(Michx Brition. Narrow-leaved Sabbatia. E angularis, (L) Pussh. Square-stemmed Sabhatia. A. calycina, (L,w) He'ler. Coast Sabbatia. E. stejlaris, Pursh. Marsh sabbatia. E. Elliottii, steurl. Ellion's Sabhatia. E. anil C. gracilis, suli-b. Slender Marsh Sabbatia E. chlor jites, Pursh. Large Marsh Sabbatia. E.

Gextaxa quinqueflora, Lam. sith Crentian. WV. crinita, Froel Fringet Gentian IV. ochroleucis, Frel. Siriperl Gentian. E. and ('. Elliottii, Chapm. Elliott's fientian. E and C. Saponari , L. Sapmont Cientian. A. Andrersii, Griseh. Closed Cientian. W. flavida, Gray: Yollowish Gemian. E. angustifolia, Wichx. One-flowered Gentian. E. 
Frasera Carolinensis, Walt. American Columbo. A.

Obolaria V̈irginica, L. Pennymort. C.

Bartoria Virginica, (L.) B. S. P. Yellow Bartonia. E. verna, (Michx.) Muhl. White Bartonia. E.

Order S:

MEXYANTHACEAE. BUCKDEAN FAMILY.

I.mмхтнемuм lacunosum (Vent). Griseb. Floating Heart. E. aquaticum (Walt), Britton. Larger Floating Heart. E.

\section{Order $<9$.}

APOCY.XACEAE. DOGBANE FAMILY.

Amsoria Tabernaemoutana, Walt. Ansonia. C. and Wr. ciliata, Walt. Pale Amsonia. C. and $\mathbf{W}$.

Tixca minor, L. Small Periwinkle. A.

major. Large Periwinkle. A.

A pocrum androsaemifolium. L. Bitter-root. A.

(anuabinum, L Indian Hemp. A

cannabinum glaberrimum, D. C. Glabrous Hemp. A.

Echitr: difformis, Walt. Echites. C.

\section{Order (ii).}

ASCLEPIADACEAE. MILKWEED FAMILY.

/A-clepias tuberosa, L. Butter-fly Ween. A.

decumbens, $L$. Decumhent Butter fly Weed. A.

lanceolata, Walt. Few flowered Milkweed. E.

rubra, L. Red Milkweed. E. and C.

laurifolia, Michx. Red Milkweed. IV.

purpurascens, L. I'urple Milkweed. II.

incaruara. L. Swamp Milkweed. A.

pulchra, Ehrlı. Hairy Milkweed. A.

obtusifolia, Michx. Blunt-leaved Milkweed. A.

exaltata (L), Muhl. Trll Milkweed. W.

variegata, L White Milkweed. A.

quadrifolia, Jacq. Four leaved Milkweed. C. and W.

Syriaca, L. Common Milkweed A.

perennis, Walt. Thin-leaved Milkweed. C.

verticillata, L. Whorled Milkweed. A.

amplexicauli-, Michx Clasping Milkweed. E.

tomentosa, Ell. Villous Milkweed. E.

Acerates viridiflora (Raf.), Eaton. Green Milkmeed. A.

viridiflora Ivesil, Britton. Ires' Milkweed. A.

lnugifolia, Ell. Furida Milkweed. E. and C.

Podostigma pubescens, Ell Purustigma. E.

Sextera maritima Decaisne. Sentera. E. 
Goxobolus macrophyllus, Michx. Large-leaved Tegro-vine. C. and W.

suberosus, R. Br. Coast Negru vine. E.

hirsutus, Miclix. Hairy Negro-vine. A.

obliquus, R. Br. Large flowered Negro-vine. E. and C. Carolinensis, R. Br. Carolina Negro-vine. A.

Orier 91.

OLEACEAE. OLIVE FAMIIY.

SYrRinga vulgaris, L Lilac. C.

Usmanthus Americanus, B. \& H. ()smanthus E.

C'hionaxthus Virginica, L. Fringe Tree. A.

Fraxinus Americana, L. White Ash. A.

viridis, Michx, Green Ash. E. and C.

pubescens, Lam. Marsh Red Ash. C.

platycarpa, Michx. Water Ash. E.

nigra, Marsh. Black Ash. E.

Biltmoreana, Bead!l. Biltmore Ash. WV.

Order 92.

HYDROI'HYLLACEAE. WATER-LEAF FAMILY.

HrDROPHYLLUM Virginicum, L. Virginia Water-leaf. W. macrophyllum, Nutt. Large-leaved Water-leaf. W. Canadense, L. Broad-leaved Water-leaf. WV. appendiculatum, Michx. Appendaged Water-leaf. IV.

Nevophila microcalyx, Nutt. Nemophila.

Phacelia bipinnatifida, Michx. Loose-flowered Phacelia. W'. parviflora, Pursh. Small flowered Phacelia. IV. hirsula, Nutt. Hairy Phacelia. W.

Purshii, Buckl. Pursh's Phacelia. W.

fimbriata, Michx. Fringed Phacelia. $W$. Hrdrolea quadrivalvis, Walt. Hairy Hydrolea. E.

Order 93.

BORAGINACEAE. BORAGE FAMILY.

Heisotropium Europaeum, L. European Heliotrope. C. Curassavicum. L. Sea side Heliotrope. E. Indicum, F. Indian Heliotrope. E. and C. anchusaefolium, Poir. Heliotrope. E. and C.

Cryogrossus officinale, L. Hound's-tongue. W.

Virginicum, L L. Wild Comfrey. IV. Lappula Virginiana (L.), Greene. Yirginia Stickseed. WV. Mretessia Virginica (L.), D. C. Virginia Cowslip. A. Irosotis laxa, Lehm. Forget-me-not. IV.

Virginica (L), B. S. P. Spring Scorpion-grass. C. Lithospermum arvense, L. Corn Gromwell. A. 
Lithosperutu latifulium, Michx. Am rican Grommell. C.

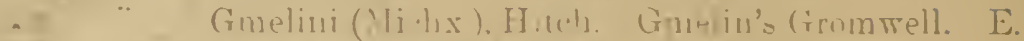

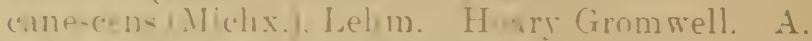

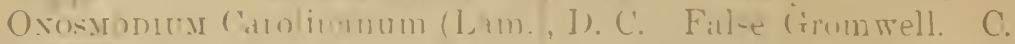
‥ Virginimmm L.). I). C. Tiruinia False Gimmmell. ('.

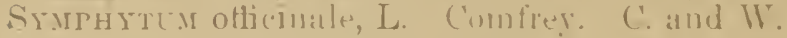

Bokrago officinalis, I. Borage. ('.

Lrcopsis arveusis, L. Small Bugloss. E and C.

Echum volgare, L. Tiper's Bugloss. C.

Order :14.

ACANTHACEAE. ACANTHUS FAMILY.

Ruelua hiflora, L. Pine-barren Ruellia. E. strepens, L. Smooth Ruellia. C. strepeus micrantba (Englm.) Britton. Ruellia. C. strepens cleistantha, Gray. Ruellia. C. ciliosa Pursh. Hairy. Ruellia. A ciliosa parriflora (Nées) Britıon. Hairy Ruellia. A. ciliosa ambiuna, Gray. Hairy Ruellia. A.

Diaxthera Americana, L. IVater Willow E. ovata, ITalt. Looseflowered Water Willow. E. and C. Jestrera brachiata Pursh. Justicia. E. and C. Calorhaxes oblongifolia, Dow. Calophanes. E.

\section{Order 9.5.}

PHRYMACEAE. I.OPSEED FAMILY.

Phryan Leptostachya, I. Lopseed. C. and $\mathrm{IT}$.

Orier 96.

\section{VERIENACEAE VERVAIN FAMILY.}

Tereera officinalis, J. Earopean Terrain. A. urticifolia, J. Nettle leaver Verrain. A. riparin, Rat. Iiver-bank Tervaiu. $\mathbb{I V}^{*}$. loastata, L. blue Vervain. A. pinnatifila, Jam. Pin nate line V'ervain. A. angustifolla, Michx. Narrow leaved Tervain. C. bracteosa, Miclux. Lnrguractel Tervain. C. Aubletia, Jacq. Lirge-flowered Tervain. E LnPIA lanceolata, Michs. Forrfmit. E. undiflora, Miclux. Figfruit. E.

- Callicalipa Americana, I. French Mulberry. C. and IV.

\section{Order $9 \%$.}

I.AIIATAE. MINT FAMILI.

Teucrium Canadense, L. H'ond Sige. C. and $\mathbb{H}^{\top}$. Ocrutur Basilicum, Id. Sweet Basil. C.

IsAnthur coeruleus, Michx. False Pennyroyal. C. and $\mathrm{IT}^{r}$. 
Taichostema dichoomum, L. Bastard Pennyoyal. ('. and W'. line alre. Nult. Nurrom Bastard Peims royal. E.

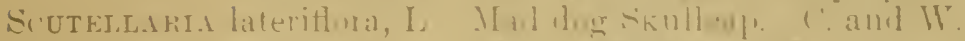

serrata, Andr. Showr skal up. C. and $\mathbb{l l}$.

cant-cme, Nutt. Downy skulcan. ('. and WT.

vers color, Nutt IItart.leared skulleap. A.

rersiculor minor: ('hapm. Heart leaverl Stalloap. A. pilosa, Michx. Hain! skulcap. WI.

integrifolia, L. IIsssop Skulleap. A.

parvula, Michx. small skull(ał. E.

campestric, Britton. Prairie Stulleap. II.

saxatilis, Riddell. Ruck skullcap. IT.

galericulata, L. Marsh Skullcap. W'.

nervosa, Pursh. Teined Skullcap. W.

Marrueium rulgare, L. Hoarbound. A.

Lophanth

scrophulariaefolius, Benth. Figwort Hyssop. W.

Cedronelia cordata, Benth. Cedronella. W.

Nepeta Cataria, L. Catnep. A.

Glechoma, Benth. Ground Ivey. A.

Prunelda vulgaris, L. Heal all. A.

Physosteria Tirginiana (L.) Benth. False Dragun-head. IV.

Virginiana sneciosi, small Dragon-head. C. and $\mathbf{W}$.

denticulata (L.) Britton. Lion's Heart. W.

SrNax̃de hispidula (Michx) Britton. Synandra. WT.

Galeopsis Tetrahit, L. Hemp Nettle. A.

Leosurus Cardiaca, L. Mothermort. A.

LAmum amplexicaule, L Dearl Nettle. A.

maculatmm, L. Spottel Derul Nettle. C.

album, L. White Dtant Tetlle. C.

Stangs hyssopifuli Michx. Hy-an! Helge Nettle. W. ambigu (Gray Brition. Dense-flnwered Herlge Nettle. IT. tenuifolin, Willi. Smonth Helge N-ttle. W.

aspera, Michx. liough IIerlge Nettle. II.

cordata, Rimbell. Crirdale Hedge Nettle. II.

arven-s, L. Corn Woumlwort. E.

Salvia lyrata. L. Lyre leareil sige. 1.

urticifolia, L. Nellle leared sage. A.

Sclarea, L. Clary Sige C.

coccinea, L. Scirlet Sige. C.

obovata, Ell. Ohovate sige. A.

officinalis, L. Crinten sige. C.

Mnsarda didyma, L. Rel Mountain Mint. W.

Clinoporlia, L. Bisil Balm. WT.

fistulcsit. L Wild Bergamot. W.

media, Willd. Purple Bergamot. II.

punctata, L. Horse-mint, C' and $\mathrm{IV}^{\prime}$.

Buephilia ciliata (L.). Raf. Downy Blephilia. C. and IV.

hirsuta (Pursh.), Torr. Hairy Blephilia. IW. 
Hedeuma pulegioides (L., Pers Pennyroyal. A.

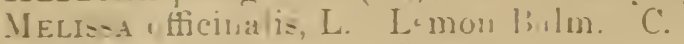

SATLrela hortensir. L Sarory C.

Calanistha Chmoporlium. Beinh. Basil weed. C.

Nepela, L. d H. Field Balm. C.

Caruliniana, Sweer. Carolina Balm. C.

Hrsiopto officinali-, L. Hyseop. C'.

Origavear majonnm, L. Maijnram. C.

Prcsanthenum limfulium, Pursh. Narrow leared Mountain Mint. $C$ and $W^{\circ}$.

lancenlatum, Pursh. Tirgivia Mountain Mint C. and 11 .

clinopodioides, T. \& G. Basil Mountain Mint. WT. hyssopifolium. Benth. Hyssn! Mountain Mint. C. and $\mathrm{W}$.

aristatum, Michx. Awned Mountain Mint. C. and W.

aristamin hy:sopifolium, Gray. C and W. incanum, Michx. Hoary Mountain Mint. C. and W. Tul ia, Benth. Southern Mountain Mint. C. and W. 'Tullia dubium, Gray. Mountain Mint. W. albe:cens, T. \& G. White-leaved Mountain Minc. C. muticum, Michx. Short-toothed Mountain Mint. E. and C.

montanum, Michx. Thin-leaved Mountain Mint. IV.

leptodon, Benth. Mountain Mint. W.

Beadlti, Small. Beadle's Mountain Mint W.

Torrevi, Beuth. Torrey's Mountain Mint. W.

Cusilla Mariana, L. Dittany. W.

Lrcopt: Tirginicus, L. Buglt-weed. A.

sessilifulius, Gray. Sessile Bugle-reed. E.

rubellus, Moench. Stalked Bugle-weed. E. and C.

Americanus, Muhl: American Bugle-weed. A.

Europaeus, L European Bugle-reed. E.

Mextha spicata, L spearnint. C. and W'.

piperita, L. Peprermint. A.

rolundifulia (L), Huds. Round-leaved Mint. A.

aquatica, L Water Mint. E. and C.

arvensis, L. Corn Mint. E. and C.

Canademis, L. American Wild Mint. C.

Colmisoovia Canadensis, L Horse-balm. A. punctata Gray. Horse balm. E.

scabriuscula, Ait. Horse balm. C.

anisata. Pursh. Horse-balm. E. and C.

HYPTI-radiata. Wllld. Hyptis. E. aud C.

Macbridea pulchra, Ell. Macbridea. E. 
Order 98.

PLANTAGINACEAE. PLANTAIN FAMILY.

Plantago major, L. Common Plantain. A.

- Rugellii, Dec. Rugel's Plantain. A.

lancenlota, L. Suake Plantain. A.

sparsiffora, Michx Southern Plantain. E. and C.

cordata. Lam. Water Plantain. E. and C.

aristata, Michx. Large-bracterl Plantain. E. and C.

Virginica, L. Dwarf Plantain. A.

pusilla, Nutt. Slender Plantain. E.

heterophylla, Nutt. Many-seeded Plantain. E. and C.

Order 99.

ARISTOLOCHIACEAE. BITTERWORT FAMILY.

Asarum Canadense, L. Wild Ginger. W.

reflexum, Bricknell. Wild Ginger. W.

Virginicum, L. Heart-leaf. A.

Virginicum grandiflorum, L. Heart-leaf. W.

macranthum (Shuttle), Small. Large heart-leaf. W.

arifolium, Michx. Halberd-leaf Heart-leaf.

Aristolochia Serpentaria, L. Virginia Snakeront. A.

Sipho, L'Her. Dutchman's Pipe. W.

tomentosa, Sims. Woolly Pipe-vine. W.

Order 100.

PHYTOLACCACEAE. PUKEWEED FAMILY.

Phytolacca decandra, L. Pokeweed. A.

Order 101.

AMAKANTACEAE. AMARANTH FAMILY.

Amarantuus retriflexus, L. Rough Pigweed. A. hybridus, L. Slender Pigweed. A.

paniculatus, L. Panicled Pigweed. A.

spinosus, L. Spring Pigweed. A.

albus, L. Tumble-weer. A.

lividus, L. Purplish Amaranth. A.

deflexus, L. Low Amaranth. A.

pumilus, Raf. C rast Amaranth. E.

Acnida cannabina, L. Salt Water hemp. E.

resocarpa, Michx. Silt Water-hemp. E.

Order 102.

CHENOPODIACEAE. GOOSEFOOT FAMILY.

Chenopodium alhum, L. Lamb's Quarters. A.

album viride (L), Moq Green Quarters. A.

glaucum, L. Oak-leaved Goosefoot. A.

Boscianum, Moq. Bosc's Goosefoot. A. 
Chexopodicy murale, L. Nettle-leared Goosefoot. A. ambrosioides, L. Mexican Tea E. and C. authelminticum. L. Wnrmseed. A. multifidum, I. Cut-leared Goosefoot. E Atriplex hastata, L. Hrlberd leaved Orache. E. a arenaria, Nult. Sea-beach Orache. E Salicoria berbacea, L. Slender Samphire. E.

Bigelovii, Tow. Bigelow samplire. E. amhigua, Michx. Wondy Samphire. E. Suated linearis, Muq. Sea Blite. E. Salsola kali, L. Saltwort. E.

Order 103.

POLSGOIACEAE. BLCLWHEAT FAMILY.

Rumex Acetosella, L. Shefr Sorrel A. hastatulus, Muhl. Engelmann's Sorrell. E. salicifolius, Wein. Willow ltared Dock. E. verticillatus, L. Swamp, Dock. E crispus, L. Yellom Dock. A. conglomeraius, Murr. Smail Green Dock C. sanguineus, L. Red-reinte Dock. E. pulcher,L. Fiddle Dock. E obtusifolius, L. Bitter Dock. A persicarioides, L. Golden Dock. E.

Fagopyrcm esculentum, Moluch. Buckwheat. W.

Polygoxum emersum (Michs.), Britton Swamp Persicaria. A. incarnatum, Ell. Pınk Persicaria. C.

Penueylyanicum, L. Pennsylrania Persicaria. C.

Persicaria, L Lady' Thumb. A.

setaceum, Baldw. Bristly Persicaria. W.

brdropipernides, Michx. Wild Water Prpper. A.

Hydropiper, L Smart reed. A.

punctalum, Ell. Water Smart-wred. C.

punctatum robustior. Small. Waler Snart-reed. E. orientale, L. Prince's Feather. C.

Tirginianum, L. Tirginia Knot-reer. W.

a riculare, L. Door-wfed. A

maritimum, L Seaside Knot-weed. E:

tenue, Michx. Slender Knot-Wred. W.

Convoloulus, ]. Black Bind-meed. A.

cilinode, Michx. Fringer Bind-weed. W.

scaudens, L Climbing False Buckwheat. A.

cristatum, E. \& G. Cresterl False Buckwheat. A.

sagittatum, L Arrow-leaverl Tear thumb. C and W. arifolium, L. Halberd lested Tear-Thumb. E. and C. birsutum, Walt. Hairy Knot-reed. E.

Porygonella parrifolia, Michx. Coast Joint-weed. E. articulata (L.), Meisn. Joint-reed. E. 


\section{Order 1iif.}

LAC'RACEAE. LAC'REL FAMILY.

Persea Caroliuensis, Nees. Red Bay. E. pubescens (Parsh), Sarg. Simamp Bay. E

SAssafras officinale, Nets. Sissafras. A.

Litsed geniculata, B it. Poud spice. E.

Lividera Bevzoin, Blume. Spicm-mond. A.

melissaffulia, Blume. Hairy Spicerrood. A.

Order 105.

THYMELEACEAE. MEZERECM FAMILY.

Dircit palustris, L. Leather-wood. E. and C.

Order 106.

SANTALACEAE, SANDALWOON FAMILY.

Comasida umbellata (L.). Bastard Trad flax. A.

Darbia umbellulata. Griy. Darbya. C.

Prrularia oleifera. Gray:" Oil Nui. C. and 11.

Buckera distichophylla, Torr. Buckleya. IV.

Order 10 ;

LORANTHACEAE MISTLETOE FAMILY.

Phoradexdrox flavescens, Nutt. Mistletoe. A.

Order 10 s.

SALRURACEAE. LIZARD'S TAIL FAMILY.

SAtrotes ceruuus. L. Lizard's-tail. A.

Order 109.

CERATOPHYLLACEAE. HORTWORT FAMILY.

Ceratophylum demersum, L. Hurnmort. E. and C.

Order 110.

CALLITRICHACEAE. WATER STARWORT FAMILY,

Calitriche palustric, L. Watar Fennel E and C.

heteraphylla, Parsh. Larser Winter Frunel. E. and C.

Order 111.

POD JSTEMACEAE. HIVER WEED FAMIIY.

Podostemox Ceratophyllum, Michx. River-weed. E and C.

Order 112.

EUPHOREIACEAE. SPURGE FAMILY.

Phylasthes Caroliuensis, Walt Carolina Pisyllauchus. C. Croton glandulosus. L. Glaudular. Cruon. i: capitatus, Michx. Capitate Croion IV. mouathogrnu=, Michx. Siugle-fruited Croton. C. 
Crotosopsis linearis, Michx. Crotonopris. C. ACALYPHA ostryaefolia, Ridd. Hornbean Mercury. C. Virginica, L. Virginia Mercury. A. gracilens. Gray. Slender M+reury. C.

Tragia ureus, L. Euslera Tragia. IV. macrocarpa, Wild. Twiıing Tıagia. C. Ricixus communis, L. Casturenl Plant. A. Jatropa stimulosa. Michx. Spuigo Nettle. A. Stillisgia sylvatica, L. Queen's Delighr. E. and C. Euphorbia polygnnifolia, L. Searide Spurge. E. maculara, L Spritterl Spurge. A. hypercifolia, Gray. Larne Syonted Spurge. A. corollata, L Flonenng spurge. A. corollata angustifolla. El. Flowering Spurge. A. marginata, P'ursh. Whicemarginerl Spurge. E, W. dentata, Michx. Tootlied Spurge. C. Ipecacuanhat, L. Ipecac Spurge. W'. heterophylla, L. Trariousleaved Spurge. C Lathyris, L. raper Siunge. W.

Darlingtonii, Gray. Darlington's Spurge. W. obtusata, Pursh. Blunt lfaved Spurge. E. commutata, Engelm. Tinter Spurge. C. Cyparissias, L Cypress Spurge. E.

Order 113.

URTICACEAE. NETTLE FAMILY.

Urtica dioica, L. Stinging Nentle. C. and W. gracilis, Ait Slenter Velte. A. urens, L. Small Netlle. A.

Laportea Canadensis. Gand. Wuid Nettle. A. Pilea pumila, Rat. Clrarmeerl. A. Boehmeria cylindrica (L), Wild. False Nettle. A. Parietaria Pennsylramca, Mull. Pellitong. A debilis, Forst. Hairy Pellitury. E.

Order 114.

CANNABINACEAE. HEMP FAMILY.

Humelus Lupulus, L. Hop. W.

Order 115.

MORACEAE. MULBERRY FAMILY.

$\checkmark$ Monts rubra. L. Red Mulberry. A. a]ha. L. White Mulberry. A.

- Broussocetia papı riferı, Vent. Paper Mulberry. A. 
Order 116.

\section{ULMACEAE. ELM FAMILY.}

Ulucs fulva, Michx., Slippery Eim. A.

$\checkmark$ Americana, L. American E'm. A.

alata, Michx Whahoo Elm. A.

$\checkmark$ Planera aquatica (Walt.), Gmel. Water Elm. A.

$\checkmark$ Celtis occidentalis, L. Hackb-rry. A.

occidentalis pumilit, Pur-h. Rnugh.leaved Hackberry. A.

Missippiensis, Bose. Southern Hackberry. A.

\section{Order 11\%.}

PLATANACEAE. PLANE-TREE FAMILY.

Plartaxts occidentalis, L. Sycamore. A.

\section{Order 118.}

JUGLANDACEAE. WALNUT FAMILY.

rJuglass uigıa, L Black Walnut. A.

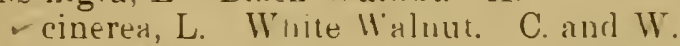

- CArra amara, Nutt. Swamp Hicku:y. C. and WV. aquatica, Nutt. Wattr Hi(kors. E. and C.

alta, Nutt. Shell bark Hiekuri. A.

tomentosa, Nutt. Whitehear Hickory C. and W.

micrucarpa, Nutt Cmall fruited Hickory. W'.

porcina, Nutt. Pigrnut Hickury. C.

sulcata, Nutt. Shag-bark Hickiry. C

Caroli, at--seprentrionalis, Ashe. Suuthern Shag-bark Hickory. C.

villosa (Sarg), Ashe. Scurfy Hickory. C.

villosa rallida, Aslie. Scurly Hicknry. C.

glabra hirsuta, Ashe. Hickuty. II.

Order 119.

CUPUIAFERAE. OAK FAMILY.

$\checkmark$ Fagus ferruginea. Ait. American Betch. A.

$\checkmark$ Castanea vesca, L. American Chestuut. C. and WV. pumila, Mill. Chinquapin. A.

pumila nana, Muhl. Larger Chinguapiu. C. and W.

$\checkmark$ Coriles.Americana. Walt. Hazel-niul. C. and W.

rostrata, Ait. B aked Hazel-nut. (' and W.

$\checkmark$ Carpinus Americana, Michx. Hombeam. A.

Ostrya Tirginica, Willd. Iron-Woud. A.

$\checkmark$ Quercus Phellos, L. Willow Oik. A

- laurifolia, Michx. Laure] O.1k. E. and C.

- inbricaria, Michx. Shingle Oak II'. einerea, Michx. High.ground Willuw Oak. E.

raquatica, Catesb. Water Oak. A.

nigra, L. Black-Jack Oak. A. 
Qunrcus Catesbaei, Miclix. Tarkey Oak. E.

- coccinea, Waug. Scarlet Oak. A.

- velutina, Lam. Black Oak. A.

- ruba, L. Ried Oak. A.

- falcata, Michx. Spanish Oak. A. falcata pagodaefolia, Ell. Spauish Oak. A. ilicifolia, Wrang. Bear Oak. C. and W. Rudkini, Britton. Rudkin's Oak. C. myrtifolia, Willd. Myrtle Oak. E.

r stellata, Wang. Pns! Oak. A. stellata parviflora, Chap. Post Oak. E. stellata margaretta, Ashe. Pust Oak. E. r alba, L. White Oak. A. lyrata, Walt. Over cun Uak. E. prinos, L. Swamp Chestnut Oak. C. Michauxii, Nutt. Michaux's Uak. E. bicolor, Willd. Swimp White Oak. W - Muhlentergii, Engelm. Chestnut Oak. C. prisoides, Willd Chinquapin Oak. A. Bladenborensis, Ashe. Bladenboro Oak. E. and C.

Order 120.

MYRICACEAE. WAX-MYRTLE FAMILY.

Mrrica cerifera, L. Wax-Myrle. E. cerifera pumila, Michx. Wax-Myrtle. E - Carolinensis, Mill. Bu-berry. E - Compronia asplenifolia, Gilert. Swelt Fern. C. and W.

Order 121.

BETULACEAE. BIRCH FAMILY.

$\checkmark$ Betula nigra, L. River Birch. A. lenta, L. Sweet Birch. W.

lutea, Michx. f. Tellow Birch. W.

Alxes viridis, D. C. Mountuin Alder. W.

, serrulata, Willd. Red Alder. A.

Order 12:.

SALICACEAE. WILLOW FAMILY.

- SAlix nigra, Narsh. Black Willow. A nigra falcata, Parsh. Narrow-leaved Willow. A. Babylouica, L. Weeping Willow. C. purpurea, L. Purble Willow. E. bumilis, Marsh. Prairie Willow. A. tristis, Ait. Sage Willow. A. discolor, Mull Hairy Willow. E.

Popults albus, L. White Piplar. A. balsamifera, L. Balsam Puplar. C. and W. 
Populus balsamifera candidans (dit.), Gray. Balm of Gilead. C. and W.

heterophylla, L. Downy Poplar. E. and C. grandidentata, Michx. Large-toothed Aspen. W. angulatus, Ait. Cottonwood. E. and C.

- monilifera, Ait. E. and C.

Order 123.

PINACEAE, PINE FAMILY.

Pixus pungens, Michx. Table-Mountain Pine. C. inops, Ait. Jersey or Scrub Pine. A.

mitis, Michx. Short-leaved Pine. A. rigida, Miller. Pitch Pine. E. and C.

r Taeda, L. Loblolly Pine. E. and C. australis, Michx. Long leaved Pine. E.

Strobus, L White Pine. IV.

Cubensis, Griseb. Pine. E.

AbIes Fraseri, Pursh. Fraser's Balsam Fir. W.

- Tsuga Canadensis (L.), Carr. Hemlock. W.

Caroliniana, Engelm. Carolina Hemlock. IV.

$\checkmark$ Picea nigra, Liuk. Black Spruce. WV.

- alba, Link. White Spruce. W.

vusiperus Virginiana, I. Red Cedar. A.

$r$ communis, L. Common Juniper. E

Taxodum distichum, Rich. Bald Cypress. E.

ThuJA occidentalis, L, Arbor Vitae. IV.

Chamaecyparis thyoides (L), B. S. P. White Cedar. E.

Taxus minor (Michx.), Britton. American Yew. IV.

\section{Class II-Endogenous Plants.}

Order 124.

PALMAE. PALM FAMILY.

1 Sabal Palmetto, R. \& S. Cabbage Palmetto. E. Adansonii, Guerns. Dwarf Palmetto. E.

Order 125.

ARACEAE. ARUM FAMILY.

Arisakma triphrllum (L.). Torr. Indian Turnip. A.

Dracontium (L.), Schnot. Green Dragnn. A. polymorplsum, Small. Dragon. IV.

Peltaxdra undulata, Raf. Green Arrow-arum. A. alba, Raf. White Arrow arum. E. 
Order 126.

LEMNACEAE. DUCKWEED FAMILY.

Lemsa polyrhiza, L. Greater Duckweed. E

trisulca, L. Star Duckweed. WV.

Valdiviana, Fhil. Valdivia Duckwred. E. minor, L. Lesser Duckweed. E.

Wolffia Columbiana, Karst. Walffia. E.

\section{Order 12i.}

TYPHACEAE. CAT TAIL FAMILY.

-Trpha latifolia, L. Broad-leaved Cat tail. A. angustifolia, L. Narrow leaved Cat-lail. E.

Sparganiug eurycarpum, Engelm. Bur-reed. C. androcladum, Engelw. Branching Bur-reed. E. and C.

Order 12s.

\section{NAIADACEAE. PONDWEED FAMILY.}

Potanogetos natans, L. Flnating Pondweed. E. and C. pulcher, Tuck. Snotted Pondreed. A.

Nutallii, C. \& S. Nuttall's Pundreeds. E. lonchites, Tuck. Ling-leaved Pondreed. E. lucens, $L$ Shining Pondwred. E. pusillus, L Small Pondweed. E. and C. hybridus, Michx. Rafinesque Pondweed. E pectinuatus. L. Fennil-leaved Pnndweed. E. and C. Pennsylvauicus, Cham. Pemsylvania Pondweed. E. aud $\mathrm{C}$.

Ruppia maritima, L Ruppix. E.

Zaunicheldia palustris, $L$ Zaunichellia. E.

NAIAs marina L Large Naia: E

flexilis, Rost. Slender Naias. E.

Zostera mariua, L. Eel-grass. E.

Order 129.

\section{ALISMACEAE. ' WATER-PLANTAIN FAMILY.}

Triglochm triandra, Michx. Three-ribbed Arrow.grass. E.

Arisma plantago, L Water Plantain. A. tenellum, Mart. Dwarf Water Plantain. C.

Echrodonos radicans, Engelm. Creeping Bur-head. A. rostratuz, Eugelm. Upright Bur-head. A.

Sagittaria calciua, Engelm. Arrow head. C. latifolia, Willd. Broad-leaved Arrow-head. A. latifolia pubescens, Muhl. Hairy Arrow-head. lancifolia, L. Lance-leaved Arrow head. E. teres, S. Wats. Slenter Arrow-head. E. graminae, Michx Grass Leaved Arrow-head. E subulata, L Subulate Arrow head. E. 


\section{Order 130.}

VALLISNERIACEAE. TAPE-GRASS FAMILY.

Elodea Canadensis, Michx. Water Thyme. W. Tallisseria spiralis, L Tape Giass. E. Limsobium Spongia Richard. Frog's-hit E.

Order 131.

BURMANIACEAE. BURMANNIA FAMILY

Buryansa biflora, L. Northeru Burmannia. E. capitata, Chapm. Southern Burmannia. E.

Order 132.

ORCHIDACEAE. ORCHID FAMILY.

CrPRIPEDIUy acaule, Ait. Stemles: Ladies Slipper. C. and IV. pubescens, Willd. Yellow Larlies Slipper. C.and W. parviflorum, Salish. Small Yelluw Ladies Slipper. C. and $W$.

spertable, salisb. Show! Ladies Slipper. W.

Orchis spectabilis, L. Shuwy Orchis. C. and W.

Habevaria orbiculata, Torr. Large Romial-leaved Orchis. IV. nirola, Sureng. Small White Orchis. E. integra, Spreng. Small Yellow Orchis. E. bractiata, R. Br. Lo' g bra'ted Or.his W. clavellata, Spreng Green Womb Orehis. W. flava, Grav. Pale Green Orchis. E. cristata, R. Br. Crested Yellow Orchi:- C. and W. ciliata, R. Br. Yellow Fringerl Orchis. E and C. finbriata, Gray. Purple Fringed Orchis. E. and C. piscodes, Gray. Small Purple Fringed Orchis. IV. blephariglottis, Torr. White Fringed Orchis. IV. blephariglottis hulupetala, Gray. White Fringed Orchis. II.

peramoena, Gray. Fringeless Purple Orchis. II.

Pogonia ophinglussoides, Ker. Rnee Pugunia. A.

trianthoghora, B. S P. Nudding Pongonis. W'.

divaricata, $\mathrm{R}$ Br. Spreading Pogonia. W.

rerticillata. Nut. Whorled Pogonia. A.

Arethusa bulbosi, L. Arethusa. W.

Spirantues cernua. Rich. Noding Ladies' Tresses. A. odorata, Lindl. Fragrant Ladies Tresses. C. and W. praecux, Kunıze. Grass-lıke Ladies' 'Tresses. E.

gracilis, Beck. Slender Ladies' Tressis. IV.

Listera convalla rioides, Torr. Twayblade. W.

australis, Lindl. South+rn Nwayblade E.

GoodyerA repens, R. Br. Ne'leaf Plantain. W.

pubescens, R. Br. Nerleaf Plantain. A.

Microstruis ophinglossioides, Nuet. Adder'smouth. E. 
LiParis liliifolia, Rich. Twayblade. IV.

Corallorhiza ndontorhiza, Nutt. Crawley-root. C. and W.

Wisteriana, Conrad. Coral-root. C.

multiflora, Nutt. Large Coral-root. C. and WV.

Tipularia discolor, B. S. P. Crane-fly Orchis. C.

Calatpogon pulchellus, R. Br. Grass-pink. E. and C.

pallidus, Clsapm. Grass-pink. E.

parviflorus, Lindl. Grass Pink. E.

Bletia aphylla, Nutt. Crested Crawley. A.

Aplectrum hyemale, Nutt. Adam and Eve. A.

Ponthineva glandulosa, $\mathrm{R}$. Br. Ponthieva. E.

Order 133.

AMARYLLIDACEAE. AMARYLLIS FAMILY.

- Amaryllis Atamasco, L. Atamasco Lily. E. and C.

r Hymenocallis lacera, Salisb. Spider Lily. E. Agave Virginiana, L. False Aloe. E and C.

Hypoxis erecta. L. Star-grass. A.

juncea, Smith. Star-grase. E.

Order 134.

HAEMODORACEAE. BLOODWORT FAMILY.

Lophiola aurea, Ker. Laphiola. E.

Aletris farinosa, L. White Colic-root. A. aurea, Walt. Yellow Colic-root. A.

Lachnanthes tinctoria, Ell. Red-root. E.

Order 135.

BROMELIACEAE. PINE APPLE FAMILY.

r'Jillandsia usueoides, L. Florida Moss. E.

Order 136.

IRIDACEAE. IRIS FAMILY.

$\checkmark$ IrIs versicolor, L. Blue Flag. A.

Caroliniana, Watson. Caroliua Blue Flag. E.

tripetala, Walt. Blue Flag. E.

Virginica, L. Virginia Blue Flag. E. and C.

verna, L. Dwarf Blue Flag. A.

cristata, Ait. Dwarf Iris. W.

Germanica, L. Fleur.de-lis. C.

1 Ixia Chinensis, L. Blackberry Lily. C. and W.

Sisyrinchium anceps, s. Wats. Blue-eyed Grass. E. and C.

Atlanticuin, Bicknell. Eastern Blue-eyed Grass. E.

- angustifolium, Mill. Pointed Blue-eyed Grass. A.

Carolinianum, Bicknell. Carolina B! ue eyed Grass. W.

1 rufipes, Bicknell. Blue eyed Grass. E. and C. scabrellum, Bicknell. Blue-eyed Grass. W. 
Order $13 \%$.

DIOSCOREACEAE. YAM FAMILY.

Droscorea villosa, L. Wild Yam. A.

Order 138.

SMILACEAE. SMIAAX FAMILY.

Syilax berbacea, L. Carrion-flower. A.

tamnifolia, Nichx. Halberd-leaved Smilax. E. and C.

ecirrhata, Wats. Upright Smilax. E. and C.

glauca, Walt. Glaucons Smilax. A.

rotundifolia, L. Horsebriar Smilax. A.

rotundifolia crenulatá, Small. Horsebriar Smilax. IV.

hispida. Muhl. Hispid Smilax. C.

Pseudo Cbina, L. Ling stalked Smilax.

Bona nox, L. Bristle Smilnx W.

rlaurifolia, L. Laurel-leared Smilax. E. and C.

Walteri, Pur-t. Walter's Smilax. E.

lanceolata, L. Larce leaved smilax. E. and C.

auriculata, Wait. Fragrant Smilax. E.

pedunculata, Muhl. Smilax. A.

Trillium sessile, L. Wake-robix. C. and W.

grandiflorum. Salish. Large Wake-robin. W.

erectum, L. Beth-ront. A.

cernuum, L. Nodding B-th-root. A.

erythrocarpum, Michx. Painted Beth.rout. A.

pusillum, Michx. Pink B-th ront. E.

stylosum, Nurt. Rose Beth root. WV.

Medeold Virgiuiana, L Indian ('ucumber-rnot. A.

Oriler 139.

LILIACEAE. LILY FAMILY.

Polygonatum biflorum, Ell. Hairy Solomon's Seal. A. gigantium, Diet. Snooth Solnmon's Seal. A.

Suilacenia racemosa, L. Large Silomon's Seal. A.

hifolia, Ker. Mountain Sulnmon's Seal. W.

stellata, L. Star-fluwered Solımon's Seal. C.

-Covvallaria majalis. L. Lily of the Valley. W.

Clistonia borealis, Raf. Yellow Clintonia. W.

umbellata, Torr White Clintonia. C. and Wr.

Allium tricoccum, Ait. Wild Leek. W

cernuum, Roth. Nodding Wild Ouion. W.

vineale, L Wiid Garlic. (?.

Canadense. L. Meadıw Grrlic. A.

¿ mutabile, Michx. Wild Oninn. A.

striatum. Jacq. Yellow False Garlic. E.

Canassia Fraseri, Torr. Wild Hracinth. W.

Erythrosium Americanum, Smith. Dog's tonth Vin'er. IV.

albidum, Nutt. Dog's-tooth Violet. IV. 
Lilium Philadelfhicum, L. Philadelphia Lily. W.

Catesbaei, Walt. Southern Red Lily. E.

Canadense, L. Wild Yellow Lily. C. and W.

superbum, L. Turk's Cap Lily. W.

Grayi, S. Wats. Asa Gray's Lily.

Carolınianum, Michx. Carolina Lily. A

Masseyi, C. W. Hyams. Massey's Lily. W.

Yucca filamentosa, $L$ Christmas-hells. $C$ and W.

gloriosa, L. Bear.grass. E.

aloifolia, L. Bear-grass. E.

Ornithogaluir umbellatuin, L. Star of-Bethlehem. A.

Muscari botryoides, Mill. Grape Hyacinth. C.

Asparagus officinalis, L. Aspraragus. A.

1 Hemerocaulis fulva, L. Day Lilv. W.

flava, L. Yellow Day Lilly. C. and W.

Order 140.

MELANTHACEAE. BUNCH FLOWER FAMILY.

Tofielda glutinosa, Pers. Glutiuous Tofieldia. W.

pubens, Michx. Viscid Tofieldia. E.

glabra, Nutt. White Totieldir. E.

Xerophyllum asphorieloides, Nutt. Turkey-beard. WV. setifolium, Small. Turkey-beard. W.

Chamahirium luteum, Gray. Star-root. A.

Aminthemum muscaetoxicum. Gray. Fly-poison. A.

angustifolium, Gray. Fly poison, E.

Stenanthium gramineum, Murong. Stenanthium. W.

Zygadenus glaberrimus, Michx. Zygadenus. E.

leimanthoides, S. Wats. Pine-barren Zygadenus. W.

Melanthium Virgi,icum, L. Bunch.flower. E. and C.

latifolium, Desr. Crisped Bunch-flower. C. and W.

parviflorum, S. Wats. Small Bunch-flower. W.

Veratrum viridi, Ait. White Hellebore. W.

Uvularia perfoliata, L. Perfoliate Bellwort. A.

sessiilifolia, L. Sessile B 1 llwort. A.

puberula, Michx. Mountaiu Bellwort.

Streptopus amplexifolius, D. C. Twisted-stalk. C. and W.

roseus, Michx. Sessile Twisted-stalk. WV.

Prosartes lanuginosa, Don. Hairy Disporum. IV.

maculata, Buckler. Mountain Disporum. W.

Pleea tenuifolia, Michx. Pleea. E.

Order 141.

JUNCACEAE. RUSH FAMILY.

Luzula campestris, D. C. Common Wood Rush. A. pilosa, Willd. Hairy Wond Rush. W.

Juxcus effusus, L. Common Push. A.

Roemerianus, Scheele. Roemer's Rush. E. 
Juscus bufonius, L. Toad Rush. A.

Gerardi, Lnisel. Blarke Rush.

tenuis, Willd. Slender Rush. A

secundus, Beaur. Serund Rush. E. and C.

dichotomus, Ell. Forked Rush. E

setaceus, Ro-1k. A wl.learid Rush. E.

marginatus, Rosth. Gras-leitral Rush. A.

repens, .lichx. Creeping Rush. A.

brachrcarpus, Enge!m Short-fruied Ru-l, C.

polycephalus, Michx. Mamy-headed Rush. E

scirpoids, Lam. Soripu=lik: Rush. E. and C.

megaceptialus, Curris Camlina Rush A.

Canadensio, Gas. Canarla Rush. A.

Canadensis subicaudatus, Engel. Canarla Rush. F RA.

Canadensis longecaudatus, Emgel. Canda Rush. A.

acuminatus, Murlix. Sharrfuilerl Rush A.

acuminatus debilis, Engelm. Sharp fruiterl Rusb. A.

Elliotrii, Chapm Ellion'- Rush. E

caudatus. Chapm. Rigid Rush. E.

asper, Engelm Rush. C. and W.

Order 142 .

PONTEDERIACEAE. PICKEREL-WEED FAMILY.

Pontederia corilata, L. Pickerti-weed. r'and IV.

cordata laneifolia. Moronse. Nirrow Pickerel-weed. C.

Heteranthera renifurmis. R. \& P. Mud Pantain. A.

graminta. Valul. Water Sar-grass. E

Order 143 .

COMMELYINACEAE. SPIDERWORT FAMILY.

Commelyan mudiflora, L. Creeping Day flower. E.

Virginica. L. Virgima Diy-flower. A.

hirtella. Tahl. Beamen Day flower. E. and C.

erecta, L. Somler Dily-flower. E.

Tradescantia Virginica, L. Sunderwnit. A.

pilnsi. Lehm. Zigzag Spiderwort. A.

rosen, Vent. Ruspanespil terwort. E.

Order 144 .

MAYACACEAE. MIYACA FAMILI.

MaYaca Michauxi, S. \& E. Maraca. E.

Order 145 .

IYRIDACEAE. YELLOW EYED GRASS FAMILY.

Xrris flexunsa, Muhl. Slender Yellnw-eyed Gra-s. A. difformis, Chapm. Soulhern Yellow-eyerl Grass. E.

Caroliniana, Walt Carolina Yellow eyrd Grass. E. 
Arks fimbriata, Ell. Fringed Yellow.eyed Grass. E. torta, Smith. Twisted Yellow.eyed Grass. E. and C. brevifolia, Michx. Pine harren Yellow-eyed Grass. E. ambigua, Beyr. Rigid Yellow-eyed Grass. E. Baldwiniana, $R$ \& $S$. Baldwin's Yellow-yed Grass. E.

Order 146.

ERIOCAULONACEAE. PIPEWORT FAMILY.

Eriocallox decangulare, L. Ten-angled Ripewort. A.

gnaphalodes, Michx. Fiattened Ripewort. A.

Paepalanthus flavidulus, Kunth. Paepulanthus. E

Lachrocallox Michauxii, Kmmb. Hairy Pipewrt. E. and C.

Order $14 \%$.

CYPERACEAE. SEDGE FAMULY.

- Crpencs flavescens, L. Yelluw Cyperus. A. diaudrus, Torr. Lur Ciperus. A. rivularis, Kunth. Shining Cyperus. C. and IV. microdontus, Torr. Coast Ciperus. E.

flavicomus, Michx. Elegant Cyperus. E. and C. inflexus, Muhl. Awned Cyperus. E and C. compressus, L. Flat Cyperus A. calcaratus, Ners. Marsh Cyperus. E. Haspan, L. Sheated Cyprus. E dentalus, Torr. 'Touthed Cyperus. C. rotundu=, L. Nut grass Ciplerus. A.

esculentus, L. Vellow Nut gra:s Cyperus. A. esculentus angustispic.tur. Britoin. Yellum Nut.grass

Cyperus. A

erythrorlizos, Muhl. Reri-ruterl Cyperus. C. speciu=us, Tahl. Michaux's Crperus. E strigosus, L. Straw-colored Crperus A strignsus capitatus, Boeckl. Straw-colnrel Cyperus. A. strigosus robustior, Kunth. Siraw colorel Cyperus. A. refractus, Engelm. R. flexed Civerus. II retrofractus (L.), Torr. Rough Cyperus. A Lancastriensis, Porter. Lincaster Cyperus. A. cylindricus, Ell. Pin binren Cyperus. E. ovularis, Torr. Globose Crinens. C. filiculmis, Vahl. sleuder Cyprrus. C. Grayi, Torr. Gray's Cyperus. E. echinatus, IIond. Balitwin's Cyperus. A. pulystachyus, Rotth. F. and C. stenolepis, Torr. E. and C. tetragonus, Ell L. distans. L. E. and 'C. virens, Michx. Cyperus. E. 
Krllisata pumila, Michx. Kyllingia. E and C.

Dulichius spathaceum. Ricb. Dulichium. E. and C.

Hemicarpa sub-quarrisa, Nees. Hemicarpa. E and C.

Lipocarpa maculata, Torr. Lipocarpra. E.

Furexa squarrosa, Michx. Squarrost Fuirena, E

hispida, Ell. Hispid Fuirena. E.

Eleocharis equisetnides, Torr. Knotted Spike rush.

quadraugulata, R. \& \& Qnatrangular Spike-rush.

ochreata, Steud. Pale Spike rush. E.

olivacea, Turr. Bright green Spike-rush. E

capitata, R. Br. Capilate Spike-rush. E. and C.

palustris, $R$ Br. Creeping Spike rusb. E. and C.

acicularis, R. \& $S$. Needle spike rush. E. and C.

tortilis. Scluultes. Twisterl Spike-rush. F.

tuberculosa, K. and S. Tubercled Spike-rusb. E.

microcarpa, Torr. Small-ruited Spike-rush. E.

melancarpa, Torr. Black fruiter Spike rush. E.

albida, Torr. White Spike rush. E.

tricostata, Torr. Three-ribherl Spike-rush. E. and C.

tenuis, Schultes. Slender Spike-rush. E.

acuminata, Nees. Flat-stemmed Spike-rush. E.

rostella, Torr. Beaked Spike rush. E. and C.

prolifera. Torr. Spike-rush. C.

pygmaea, Torr. Spike-rush. E.

Scirpes caespitosus, L. Tufter Club ru-h. W.

Clintoni. Gray Clinton's Club-rush

debilis, Pursh. Weak stemmed Club-rush, E. and C.

pungens, Vahl. Three-square Ciuh-rush. C.

Olneri, Gray. Olner's Bulrush. C.

leptolepis. Charm. Canby's Bulru=b. E.

lacustris, L. Great Bulrush. A

sylvaticus, $L$. Wond Bulrush A.

atrovirens, Muhl. Dark-green Bılru-h. C and W.

polyphyllu-, Tahl. Leafy Bulrush. C

Eriophoruir Tirginicum, L. Tirginia Colton-grass. E.

Fimbristylis spadicea, Vahl. Sliff Fimbri=lylis. E.

sparlicea puberula, Chapm. Fimtristylis. E.

rastanta, Tahl. Marsh Fimbritylis E.

laxa, Tahl. Weak Fimbristylis. A.

Tahlii. Link. Tahl's Fimhristri- IV

autumnalis, R. \& S. Slender Fumbristylis.

A.

IsoLEPIs capillaris, R. \& $\mathrm{S}$. Isolepi: E

ciliatifolia, Torr. Isolepis. E.

steunphylla. Torr. Isolepi- E.

Rhyschospora corniculata. Gray. Hormed Rush E. corniculata macr's'achira (Turr), Britton. E.

pallida, Curtis. Pale Beaked-rush. E.

oligautha, Gray. Few-flowered Beaked-rush. E. aud $\mathrm{C}$. 
Rhrenospora alla (L.), Vahl. White Beaked-rush. A. glnmerata (L), Talil. Clustered Beak-rush. E.and C. Flomerata naniculata (Gray). Chapm. Clustered Beak-ru-h. E and C: glomra'd discutiens, Clarke. Clustered Beakedrush. E. and C. cephalautha, Gray. Capitati Berked-rush. C. gracilenta. Gruy: Slender Beaked-rush. E. crnmsa, Ell Gress like Beaked-rush. E. and C. Torr rall: Gray. Torrey's Beaked-rush. E. inex lansa (Michx.), Yahl. Nodding Beaked-rush. A. plumusa, Ell. Beaked rush. $\bar{E}$.

micrucarpa, Baldw. Beaked-ru-h. C. miliacea, Gray. Beaked-rush. E. aud C. Grayii, Kunth. Grar's Beaked-rush. E. m+galecarpa, Gray. Beakes-rush. E. (illata, Tahl. Béaked-rush. E. fa-cicularis. Nutt. Beaked rush. E. fu-coidts, Bolkl. Beaked-rush. E. tilifulia, Grav. Beaked rush. E.

Psilocaria nitens (Vahl.), Wrod. Short-beaktd Bald-rush. E.

Dichromexa Leucoc-phala, Michx. Narrow-leaved Dichromena. E. latifulia, Buldw. Broadleaved Dichromear. E.

Cladilm mariscrides, Torr. I wig-rush. A. effusum, Torr. Saw'grass. A

Scleria oligantha, Michx. Frw-flowertd Nur-rush. E. triglomerata, Michx. Tall Nut-rush. E. and C. veticularis, Michix. Railulater Nutrush. E. veticularis pubescens. Brittou. Pubesceut Nut-rush. E. veticularis itscura, Brition. Obscure Nut-rush. Torrevana, Waly. Torrey's Nut-rush. E. pauciflora, Muhl. Papillose Nut-ruslı. E verticillata, Muhl. Luw Nut-rush. E. and C. Elliottii, Chapm. Ellintt's Nut-rush. E.

Carex Collinsii, Nunt. Collius' Sedge. E intumescens. Ruilge. Blailder Sedge. E and C. Asa Grayi, Bailey. Gray's Sedge. E. and C. grandis, Bailey Large Srdge. C. bullata, Scuk. Button Serlge. A. lurida, Tahl. Sullow Sedge. E and C. Jurica flaccida, Briley. Sallum Sedge. C. Frankii, Knutc. Frank's stdge. E. squarrosa, L. Squarrose S.dge. E. typhinoidrs, Schwein. Cat-tail Serige. E. and C. scabrata, Schwein Rough Serdge. W. vestity, Willd. Velvet sellge. C. Walteriana, Bailey. Wiltrr's Selge E. torta, Buott. 'Twisted Stdge. C. and W. 
CAREx prasina, Wahl Drooping Serge. IT littoralis, schwein. Barratt's Sedge. E. gynandra, Schwein. Nodding Sedge. WV. virescehs, Muhl. Downy Green Sedge. C. and W. Costellata, Britom. Ribibed serlge. WT. triceps, Michs. Hirsute Serige. C. and IV. Caroliniana, Schwein. Carolina Sedge. C. gracillima, schwein. Graceful Sedge. C. aestivalis, Curis. Summer Sedge. IV. tenuis Rudge. Slender-stalkerl Sedge. W. oblita, Steud. Dark-green Sedge. C. grisea, Wahl. Gray Sedge. A. amphibola, steud. "Narrow-leaved Sedge C. flaccosperma, Dewey. Thın-fruited Sedge. IV. polymurpha, Muhl. Tariable Senge. C and W. tetanica, Schk. Wrod's Sedge. E. aud C. Mearii, Dewey. Mrad's Sedge. E. laxiflora, Lam. Loose flowered Sedge. IT. laxiflura patulifolia, Carey. Serlge. II. blanda, Dewey. Sedgr. IT. styloflexa, Buckley. Bent Serdge. C. digitalis, Willd. Wond Srdge. C. aud W. ptychocorpa, Steud. Thicket Sedge. C. pedicellata (Dewey), Britton. Fibruus-routed Sedge. IV. Pennsilrainca, Lam. Penu-rlrania Sedge. IV. nigro-margierata Schweiı. Black edged Sełge. C. Fraseri, Andr. Fra-er's Serge. IV. leptalea, Wahl. Bristle stalked Sedge. W. stipatu, Muhl Awl-irnited Serige. C. aud WT. vulpinoidea, Michx. Fox sedge. C. rosea, schk. Stellati Sedge. C. and IT. rosea radiata, Dewer. Stellari Sedge. C. and W. cephalaphora, Muhi. Oral-headed Serge. C. Mitchelliana, Curtis. Mitchell's Sedge. C. bromoides, schk Brom like Serge. E. decomposeta, Muhl. Large-paniclerl Sedge. C. retroflexa, IIuhl. Reflextd Sedge. IV. tribuloidts, Wahl. Blunt broom Sedge. IV. tribuloides Bebbii. Bailey Blunt hrum sedge. IV. straminea, Wild. Straw Sedge. W. straminea foenea, Torr. Siraw Sedge. IV. straminea miratilis, Dewey. Straw Sedged. W. alata, Torr. Broad-winger Serge. E. crinila, Lam. Fringed Sedge. A. gynandra, Schwein. Norlding Serge. E. Willdenovii, Schk. Willdenow's Sedge. IT. fusca, All. Brown Sedge. WT. Davisii, S. \& Torr. Davis' Sedge. W. 
Carex conoidea, Schk. Field Sedge. W. oligocarpa, Schk. Few-fruited Sedge. IT. plantaginea, Lam. Plantain-leaved Sedge. WT. folliculata australis, Bailey. Folliced Serge. E. and C. oblita, Steud. Dark green Sedge. E. juncea, Irilld. Juncus Sedge. IV. verrucosa, Mubl. Glancous Sedge. E. turgescens, Torr. Chamrel leaved Sedge. E riparia, Curtis. River-bank Sedge. A.

Order $14 \mathrm{~s}$.

GRAMINEAE. GRASS FAMILY.

Tripsactar dactyloides, L. Gama Gras. A.

Axthoxaxthuir odoratum, L. Sweet Ternal Gras: C.

Ep.intrits alopecuroides (L), Ell. Spiral-awned Beard Grass. A. saccharoides. Michx Plume Grass. E. complactus, Nash Contracled Plume Grass. E. and C. brevibarbis, Michx. Shor beared Plume Grass. A. Stipa arenacea, L. Feather Griss E. Maxisuris rugosa, Kunize. Wrinkled Man'suris. E. granulari=, Swarlz. Manisuris. C.

Axdropogox scoparius, Michx. Bronm Beard-grass. A. argyraeus. Schultes. Silvery B Bard.grass. A. furcatus, Muhl. Furked Beard-grass. C. Virginicus, L Virginia Beard-grass. C. glomeratus (Tralt.), B. S. P. Bushy Beard.grass. E. Elliattii, Chapm. Elliot's Beard-grass. C. Chrisopogox avenaceus (Michx.), Benth. Indian Grass. A. Sorghur Halopense (L.), Pers. Johnson-grass. A. Nazia raceniosa (L.), Kunize. Prickle grass. E. Paspalum mucronatum. Muhl. Water Paspalum. E. and C. racemulosum, Nuti. C. membranaceum, Walt. Walter's Paspalum. E. purpurascens, E'l. C. distichum, L. Joint grass. A. dilatatum. Poir. Tali Paspalum. A. setaceum, Michx. Slender Paspalum. A. ciliatifolium, Michx. Ciliare leaved Paspalnm. C. ciliatifolium dasyplyyllum, Ell. Prspalum. C. longipedunculatum, LeConte. Long-stalked Paspalum.

E. and C:

rlaeve. Mich. Firld Paspalum. A.

Floridanum, Michx. Florida Paspalum. A. praecox, IIali. E. compressum (Sw.), Nees. Flat Paspalum. A. difforme, Le('onte. E. paspaloides (Michx), Scrib. Crab-grass Paspalum. A. furcatum, Flugge. Paspalum. E. 
Miliumanphicarpon, Pursh Amphicarpon. E.

Panicum consanguineum, Kath. C.

Addisonii, Nash. E.

Ashei, Pearson. Ashe's Amphicarpon. E

Scribneriauum, Nash. Scribner's Amphicarpon. E. and $\mathrm{C}$.

scuparium, Lam. C.

scabriusculum, Ell. E.

Joori, Vasev. Juor's Amphicarpon. C.

macrocarpon, Le Cumle. Amphicarpon. W.

gibbum, Ell. Gıbbons Amphicarpon. E. and C.

verrucosum, Muhl. Warty Amphicarpon. E.

capillare, L Witch.grass Amphicarpon. A.

proliferum, Lam. Spreading Amphicarpon. E. and C.

miliaceum. L. Millet. C.

amarum, Ell. Sea beach Amphicarpon. E.

Crus-galli, L. Baruyerd Graes A.

IValteri, Pursh Sali-marsh Cuckspur. E.

digitarioiles, Carpenter. Narrow Panicum. E. and C.

hians, Ell. Gaping Panicum. A.

ancep, Michx. Beaked Panicum E. and C.

agrostidilorme, Lam. Agrostis-like Panicum. E.

elougatum, Pursh. Long Panicum. A.

spharrocarnun, Ell. Round fruited Panicum. A.

microcarporm, Muill. Small funited Panicum. A.

Porterianum, Nash. Porter's Panicum. C.

commulatum, schults. Tarialile Panicum. A.

clandestinum. L. Hispid Panicum. E. and C.

laxifl rum, Lam. Lax flowered Panicum. C.

depauperatum, Muhl. Starverl Panicum. C.

angusifolinm, Ell. Narrow-leaved Panicum. A.

ut urauthum, Griseb. E.

nemopanthum, Ashe. E. and $\mathrm{C}$.

dichatonum, L. A.

dichatonum elatum, Ta.ey. C.

maculatum, Ashe. ('.

Ruauakense. Ashe E. and C.

demis-um, Triu E and C

Mattamu-ketense. Ashe. E.

erisifulinm, Ba1ll. C.

Wrightianum, Scrib. spliaguicolam, Nash.

lucidum, Astie. E.

Cuthberiii, Ashe. C.

parvispicnlatum, Nash. E.

leucothrix, Nash E

huachucae, Ashe. C.

viscidum, Ell. E. and C. 
Panicum ciliferum, Nash. E.

Atlanticum, Nash. E.

haemacorpon, Ashe. C.

arenicolum, Ashe. C.

annulum, Ashe. C.

meridionale, Ashe. C. and W.

filiculme, Ashe. C.

microphyllum, Ashe. C.

glabrissiuum, Ashe. E.

Nashianum, Scrib. E.

scrotinum, Trin. C.

laniginosum, Ell. E.

Columbianum, Scrib. C.

Setaria glauca, Beauv. Yellow Fox-tail Grass. A. viridis, Beauv. Green Fox-tail Grass. A.

Italica, R \& S. Hungarian Fox-tail Grass. C.

verticillata, Beauv. Fox-tail Grass. A.

Cenchrus tribuloides, L Hedge-hog Grass. E.

incertus, Curtis. Hedge-hog Grass. E

Zizania acquatica, L. Wild Rice. E.

miliacea, Michx. Wild Rice. E.

Leersia Virginica, Willd White Grass. E. oryzoides, Sw. Rice-cut Grass. E.

lenticularis, Michx. Catch fly Grass. E

Hrdrocloa Carolinensis, Beav. E.

Phleum pratense, L. Timothy. A.

Cinna arundinacea, L. Wood Reed. A.

latifolia, Griseb. Slender Wond Reerl. A.

Holcus lanatus, L. Velvet "r Meadow Grass. A.

DActylis glomerata, L. Orchard Grass. A.

$\checkmark$ PoA annua, L. Annual Meadow Gracs. A.

- compressa, L. Flat-stemmed Meadow Grass. A.

r pratensis, L. Kentucky Blue Grass. A.

trivialis, L. Roughish Meadow Grass. A.

flexuosa, Muhl. Flexuous Spear Grass. C.

sylvestris, Gray. Sylvan Spear Grass. C. and W.

alsodes, Grav. Grove Spear Grass. C. and W.

brevifolia, Muhl. Short leaved Spear Grass C. and W.

Glycera elongata, Trin. Long Mauna Grass. C. and W. nervata, Trin. Nerved Manna Grass. C. and W. pallida, Trin. Pale Mauma Grass. E. and C.

- Festuca Myurus, L. Rat's tail Fescue Grass. A.

- elatior, L. Meadow Fescue Grass. A.

nutans, Willd. Nodding Fescue Grass. C. and W.

Bromus ciliatus, l. Fringed Brome-grass. A.

hordeaceus. L. Soft Brome-grass. C.

secalinus, L. Cheat Brome-grass. A.

racemosus, L. Upright Brome-grass. C. 
Lolium temulentum, L. Darn+ll Grass. C.

Hordeum pratense, Huds. Meadow Barley. A.

Axthaenantia villosa, Benth. Anthaenantia. E

rufa, Benth. Purple Anthaenantia. E.

Phalaris intermedia. Bosc. Canary Grass. E.

Hierochloe borealis, R. \& S. Senec: Gra's. C.

Alopecurus geniculatus, L. Marsh Fux-tail. A.

pratensis, L Mealdow Fox-tail. A.

Sporobolus asper, Kunth Krugh Rush-gras:. A.

vaginaeflorus, Wond. Sheatherl Rush grass. A.

vaginaeflorus minor, Vasey Sheathed Rush grass W.

Virginicus, Kunth Seashore Rush-grass. E

Indicus, Brown. Smut Rush grass A.

Junceus, Funth. W'ire-grass. E and C.

cryptandrus, Gray. Wire-gras: E.

Agrostis alba, L Herd's grass. A.

- rubra, L Red Bent grass. II'.

perennans, Tuck. Thin Grass IT

scibra, Willa. Rungh. Hair Grass. A

elata, Trin. Tall-Bent Grass. E.

Novae-Angliae, Tuck. New Eugland Bent-grass. W.

canina, L .Imuntain Bent-grass. IV.

Polypogon maritimus, Willd. Baar 1 Gries. E.

Calanagrostis Canalensis, Beauv. Blue joint Grass. II.

Nuttallian:, Steur. Nuttall's Reed Grass. A.

arenaria. Roth. Samil reed Grase. E.

Aristida dichotoma, Michx. Poverty Grass A.

gracilis, Ell. Slender Poverty Grass. A.

purpurascens, Poir. Purplish Povery Grass. A.

lanata, Poir. Woolly Piverty Gras: A.

virgata, Trin. Tirgate Poverty Griss. E.

stricta, Michx. Erect Poverty Grass. E and C.

Muhlenbergia sobolifera, Trin. Rock Muhlenhergia C.

Mexicana, Triu. Meadow Muhlenbergia. A.

glomerata, Trin. Marsh Muhlentergia. C.

sylvatica, T. \& G. Wond Mulslenbergia. W.

diffusa, Schreb Drop-seed Muhlembergia. A.

capillaris, Kunth. Long-awned Mublenbergia. E.

Brachyelytrum erec um, B-auv. Brachyelirum. W.

Airalcaryophyllea, L. Silvery Hair.grass. C.

Deschanpsia flexuosa, Trin. Nary Hair-gras.. IV.

Trisetum subspicatum, Beauv. Narrow False Oat. IW.

palustris, Michx. Marsh False Oat. A.

Danthonia spicata, Beauv. Common Wild Oat-grass. A.

sevicea, Nutt. Silky Wild Oat-grass. A.

compresia, Austiu. Flattened Wild Oat-grass. W.

glabra, Nash. Smooth Wild Oat-grass. E.

Arrhenatherum elatius, Beauv. Oat-grass. A. 
Spartixa polystacha, Ell. Salt Reed grass. E. juncea, IVilld. Salt-meadow Grass. E. stricta, Roth. Smouth Marsh Grass. E. stricta glabra. Muhl. Smooth Marsh Grass. - E. stricta alteruifolia, Lnis. Smooth Marsh Grass. E Grunopogox racemosus, Beauv. Gymnopogon. E. hrevifolius, Trin. Gymnopngon. E.

Chloris petraer, Swartz. Chloris. E.

Crinodox Dactylon, Pers. Bermuda Grass. A

Ctexicm Americanum, Spreng. 'Toothache Grass. E Eleusine Egrutiacil, Pers. Crow font Grass. A.

Indica, Gaert. Crab Grass. A.

Leptociod mucronata, Kunth. Leptocloa. A.

DiplachNe fascicularis, Beaur. Diplachne. E rigida, Munru. Diplachne E

Triodia cuprea, Jacq. Tall Red top. A.

Triplasis Americana. Beaur. Triplasis. E. purpurea, Chapm. Triplasis. E.

Melica mutica, Walt. Mellic Grass. E. diffusa, Yursh. Mellic Grass. E.

Eatoria Dudleyi, Vasey. Dudley's Eatouia. IW. cobtusati, Gray Blunt Entonia. E ami C.

Pennylranica, Gray. Pennsylvania Eatonia. C.

Eragrostis reptan-, Nees. Creeping Eragrostis 'A. migastachyia, Link. Eragrostis. A. ciliaris, Link. Eragrnstis. A.

Purshii, Schrad. Pursh's Eragrostis. A. pilosa, Beauv. Tulted Eragrostis. C. tenuis, Ell. Hairy Eragrostis E and C. capillaris, Nees. Capillary Eragrostis. A pectinacea, Gray. Purple Eragrostis. C. campestris, Trin. Mearow Eragrostis.

Uxiola gracilis, Michx. Slender Spike grass. A. latifulia, Nichx. Broad Spike-grass. E and C. paniculata, L. Ser Oats. E spicata, L. Marsh Sea Oats E.

Phragaites communis, 'T rin. Reed. E. Agroproum repens. Beaur Conch-grass. A.

Caniuum, R. \& S. Conch grass. A.

Exymus robustus, Serib Stout Rye Grass. E, and C.

Virginicus, L. Trrginia Rve Grass. A.

striatus, Willd. Nodding Rye Grass. C.

Asprella Histix, Willd. Boltle-brush. C.

Arundixafia macrosperma, Michx. Cane. A. tecta, Muhl. Reed. A. 


\section{Class III-Acrogens.}

Cryptoganons or Flowerless Plants.

Order 149.

EQUISETACEAE. HORSE-TAIL FAMILY.

Equisetum laevigatum, Braun. Smooth Scouring-rush. A.

lyyemale, L. Common Scuring.rush. IV.

sylvaticum, L Wood Horse-tail. E.

arvense, L Field Horse-tail. C.

Order 150.

OPHIOGLOSSACEAE. ADDER'S TONGUE FAMILY.

Botrychium ternatum (Thimb), Sw. Ternate Grape fern. E. and C. dissectum. Granefern. E. and (.

Virginianum (L.), Sw. Virginia Grape-fern. A.

Order 151.

POLYPODIACEAE. FERI FAMILY.

$\checkmark$ Polypodium rulgare, L. Common Polypory. C. and W. vulgare cristatum. Common Polypody. W.

valgare Cambricum. Common Polypody. IV.

incanum, sw. Tree Polypody. E. and C.

Pteris aquilina, L. Rock Brake. A.

aquilina caudata (L), Hork. Rork Brake. E.

tremuloises. C.

Pellaea atropurpuria, Link Cliff-trake. IV.

Chetlanthes Alabamensis, Kuntze. Alabama Lip-fern. W.

lanosa, II'alt Hairy Lip-ferm. IV.

tomentosa, Link. WWoolly Lip-fern. W.

vadiatum Capillus-Veneris, L. Tenus-hair Fern. E.

- pedatum, L. Maiden hair Fern. A.

Woodwarda Tirginica, Smith. Tirginia Chain Fern. A. angustifolia, Smilh. Net veined Chain Fern. E. and $\mathrm{C}$.

CAmptosonus rhizophyllus, Link. Walking-fern. W. Asplexium pinnatifidum, Nutt. Pinnatifid Spleenwort. W. ebenoides, Scott Scoit's Spleenwort. IV. parvulum, $\mathrm{M} \& \mathrm{G}$. Small Spleenwort. IV. platyneuron, Odkes. Ebony. C. and IV. Trichomanes, L. Maiden hair Spleenwort. II'. angustifolum, Michx. Narrow-leaved Spleennort. IV. Ruta-muraria, L. Wall Rue Spleenwort. W. montanum. Willd. Mountain Spleenwort. WT. Bradleyi, Eaton. Bradley's Spleenwort. W. thelypteroides, Michx. Silvery Spleenwort. 
Asplexium Filix-foemina, Beruh. Ladr-fern. A. dentatum, L. Spleenwort. WW.

Crstopteris hulbifera, Beruh. Bulbons Brittle Fern. W. fragilis, Buruh. Brittle Fern. IV.

Aspidium acrustichoides, $\mathrm{S}$. Whristmas Fern. A.

"acrostichoides Schweinizii. Christmas Ferm. A.

Noreboracense, Sw. New Iork Feru. IV.

Thelipteris, Sw. Shield Fern. A.

Goldieanum, Hook. Goldie's Fern. C and IV. marginale, Sw. Wood Fern. C. and W.

spinulosum, Sw. Spinulos Shield Fern. IT.

spinulosum intermedium, Exton. Spinulose shield Fern. II.

spinulosum dilatatum, Hork. Sirinulose Shield Fern. W. Phegopteris polypodioides, Fre. Lning Becch Feru. IT.

hexagonoptera. Fee Broar Beech Fern. A.

Dryponteris. Fee Oak Fern. E. and C.

Dicksoria punctilobula (Michx). Grav. Hay-scented Fern. W.

LFGodicM palmatum, sw. Climbing Fern. A.

Oxoclea sensibilis, L. Sensitive Ferri. A.

Woodsia Ilrensis. R. Br. Rusty Woodsia. IT obtusa, Torr. Blunt-lobed Woodsia. IV.

Osmuxda cinnamomea, L. Cinnamon Fern. A.

- regalis, L. Royal Fert. A.

- Claytoniana. L Clayton's Fern. A.

\section{Order $15^{\circ}$.}

\section{LYCOPODIACEAE CLUE-MOSS FAMILY.}

Lycopodium lucidulum, Michx. Shining Club moss. IV. Selago, L. Fir Club muss. IT. clavatum, L Rumning Club-moss. W. dendroideum, ilichx. Ground Pine Club moss. IV. Carolinianum, L Carolina Club-moss. E. - complanatum, L. Christmas Club-moss. WT. innurlatum, L. B ig Club.moss. E. alopecuroides, L. Fox tail Club-moss. E alopecuroirles adpressum, Chapm. Club-moss. E.

Order 1.53.

SELAGINELLACEAE SELAGINELLA FAMILY.

Selagivella rupestris, Spring. Rock Selaginella. A. apus, Spring. Creeping Selaginella. A. Order $15 t$.

SALVISIACEAE. SALVIXIA FAMILY.

Azolla Caroliniana, Willd. Carolina Azolla. E. 


\section{INDEX.}

\begin{tabular}{|c|c|}
\hline $\begin{array}{r}\text { PAGE. } \\
\text { SHI }\end{array}$ & Autirrhinum \\
\hline 29S & Anỵchia \\
\hline Acalypha_. & 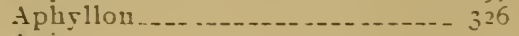 \\
\hline Acanthospermum & Apios ...... \\
\hline Acer & A pium ..... \\
\hline Acerates _........... 330 & Aplectrum ... \\
\hline Achillea & A pocynum .. \\
\hline Acnida & Apogon ..... \\
\hline - & Aquilegia ... \\
\hline 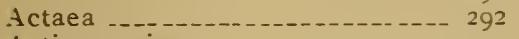 & Arabis \\
\hline Actiuomeris & Aralia ........ \\
\hline Adiatum _. & Arctium_.... \\
\hline Adlumia..... & Arenaria \\
\hline Adonis _................... $29 \mathrm{I}$ & Arethusa ....- \\
\hline Esculus _. & Argemone.............. \\
\hline 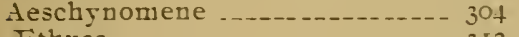 & Arisaema ............ \\
\hline Ethusa & $\begin{array}{l}\text { A } 1 \text { istida } \\
\text { Aristolochia }\end{array}$ \\
\hline $\begin{array}{l}\text { Agave } \\
\text { Agrimonia }\end{array}$ & $\begin{array}{l}\text { Aristolochia } \ldots \ldots \\
\text { Aruica }\end{array}$ \\
\hline Agropyrum & Arrhenatherum \\
\hline Agrostemma & Artemissia _- \\
\hline Agrostis _. & Arundinaria ........... \\
\hline Ailanthus -n_ & Asarum - \\
\hline Aira & Asclepias $\ldots$ \\
\hline Alchemilla _-_an & Ascyrum --- ------- \\
\hline Aletris _- & A simina \\
\hline Alisma _... & Asparagus _.................. \\
\hline Allium & $\begin{array}{l}\text { Aspidium } \\
\text { Asplenium }\end{array}$ \\
\hline $\begin{array}{l}\text { Alnus } \\
\text { Alopecurus }\end{array}$ & $\begin{array}{l}\text { Aspleminu } \\
\text { Asprella }\end{array}$ \\
\hline Alsine & Aster \\
\hline Alyssum_._. 295 & Astilbe \\
\hline Amarantus _. & Astralagus .....- \\
\hline Amaryllis ... & 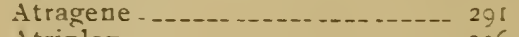 \\
\hline A nubrosia & Atriplex _._- \\
\hline Amelanchier & Atropa \\
\hline Anianthium & 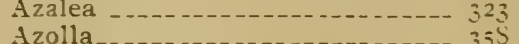 \\
\hline $\begin{array}{l}\text { Ammania } \\
\text { Amorpha. }\end{array}$ & zolla \\
\hline $\begin{array}{l}\text { A morpta } \\
\text { Ampelopsis }\end{array}$ & Baccharis .... \\
\hline Amphicorpaea & Bald winla -- - \\
\hline Amsonia _-.. & Baptisia \\
\hline ygdalis & Barbarea ................ \\
\hline Anagallis _................... 325 & Bartonia \\
\hline Andromeda & Batrachium _............. \\
\hline Andropogon _................... 352 & Bellis \\
\hline Anemone _. & Berberis \\
\hline Angelica & $\begin{array}{l}\text { Berchmeria } \\
\text { Berlandiera }\end{array}$ \\
\hline 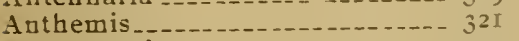 & Betula \\
\hline thenantia ................... 355 & Bidens :................... \\
\hline Authoxanthum _...... $35^{2}$ & Bigeloria___._. 319 \\
\hline
\end{tabular}




\begin{tabular}{|c|c|}
\hline Bignonia & $\begin{array}{l}\text { PAGE. } \\
-\quad 29 S\end{array}$ \\
\hline 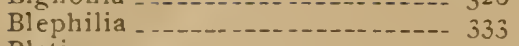 & Ceratophyllum \\
\hline Bletia ........ & Cercis . . \\
\hline Boehneria & Chaerophyllum \\
\hline Boltonia_...-.- & Chamaecyparis \\
\hline 332 & Chamaelirium \\
\hline Borrichia & Chaptalia --- \\
\hline $\begin{array}{l}\text { Botrychium - } \\
\text { Bovkinia }\end{array}$ & $\begin{array}{l}\text { Cheilanthes } \\
\text { Chelidonium }\end{array}$ \\
\hline Boykinia & $\begin{array}{l}\text { Chelidonium } \\
\text { Chelone }\end{array}$ \\
\hline asera & Chenopodium \\
\hline Issica _.............. 295 & Chimaphilla \\
\hline reweria _...... 328 & Chiogenes -- \\
\hline Bromus - & Chionanthus \\
\hline Broussouetía _- - & Chloris _......... \\
\hline $\begin{array}{l}\text { Bryophy̆lum } \\
\text { Bucknera }\end{array}$ & $\begin{array}{l}\text { themum } \\
\text { lanus }\end{array}$ \\
\hline Buckleya & $\begin{array}{l}\text { Chrysobalanus -- } \\
\text { Chrysogonum -- }\end{array}$ \\
\hline melia_............ 325 & Chrysosplenium \\
\hline Bupleurum _. & Chrysopsis_...-- \\
\hline nannia _...-. & $\begin{array}{l}\text { Chrysopogon } \\
\text { Cichorium }\end{array}$ \\
\hline Cabomba _-_-_-_. & Cicuta \\
\hline Cacalia & Cimicifuga .. \\
\hline Cakile -_-_-_-_- 295 & Cinua \\
\hline Calamagrostis _- & Circaea \\
\hline 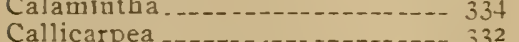 & $\begin{array}{l}\text { Cissus }-. .-1-. .- \\
\text { Cladium }\end{array}$ \\
\hline Callirrhol $\ldots \ldots \ldots$ & Cladrastis \\
\hline Caliitriche & Claytonia ...... \\
\hline Calophan $s$ _. & Clematis \\
\hline Calol,ogon & Cleome \\
\hline Caltha & Clethra \\
\hline $\begin{array}{l}\text { Calycanthus } \\
\text { Calycocarpum }\end{array}$ & $\begin{array}{l}\text { Clintonia } \\
\text { Clitoria }\end{array}$ \\
\hline $\begin{array}{l}\text { Calycocarpum } \\
\text { Camassio }\end{array}$ & $\begin{array}{l}\text { Clitoria } \\
\text { Cnicus }\end{array}$ \\
\hline Cameliua & $\begin{array}{l}\text { Cnicus } \\
\text { Cocculus }\end{array}$ \\
\hline Campanula & Collinsonia \\
\hline Camptosorus & Comandra \\
\hline Capsella & Commelyna \\
\hline Cardamine _............ 294 & Couptonia \\
\hline Cardiospernum _............ 302 & Conioselinum _..... \\
\hline Cardutus _. & Conopholis ............. \\
\hline Carex & Convalla ria ......... \\
\hline $\begin{array}{l}\text { Carphephorus -n- } \\
\text { Carpinus }\end{array}$ & 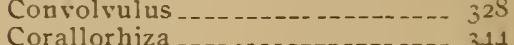 \\
\hline $\begin{array}{l}\text { Carpinus } \\
\text { Carum }\end{array}$ & $\begin{array}{l}\text { Corallorhiza } \\
\text { Coreopsis }\end{array}$ \\
\hline Carya & Coriandrum \\
\hline Cassaudra .... 324 & nus \\
\hline Cassia & Corydalis \\
\hline Castanea & Corylus ... \\
\hline Castilleia & Crantzia... \\
\hline Catalpa & Crataegus ........ \\
\hline Cancalis & Crepis - - \\
\hline Caulophyllum _-_-_- & Crotalaria \\
\hline Ceanothus & Croton - \\
\hline $\begin{array}{l}\text { Cedronella }-10 \\
\text { Celastrus }\end{array}$ & 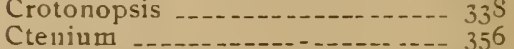 \\
\hline Celtis & $\begin{array}{l}\text { Cteminm } \\
\text { Cunila }\end{array}$ \\
\hline ichrus & Cuphea \\
\hline Centaurea & Cuscuta \\
\hline Centrosema & Cynodon \\
\hline Centunculus _. & Cynoglossum ..... \\
\hline Cephalanthus........................... & \\
\hline
\end{tabular}


Cypripedium

Crrilla - .

Cystopteris .................... $35 \mathrm{~S}$

Cytissus _...

Dactylis ........ 354

Danthonia -...-_..-_._- 355

Darbya............... 337

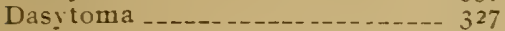

Datura _... -................... $32 \mathrm{~S}$

Daucus_._... _._.

Decumaria .................... 30S

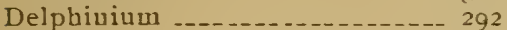

Dentaria _................ 295

Deringa ........................ 3 II

Deschampsia _._._._-_._-_ 355

Desmodium _..._._._._._._._. 304

Diamorpba _................ 309

Dianthera _................. 332

Dianthus _... _._. _._._._._._._. 297

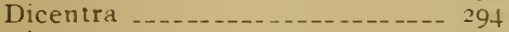

Dichromena .................. 350

Dichoudra -................ $32 \mathrm{~S}$

Dicksonia _..._.

Didiplis ........................ 309

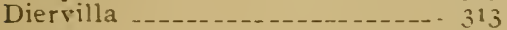

Digitalis _................. 327

Diodia _................. 313

Dionaea _._._._._._._._. 294

Dioscorea _..._................. 345

Diosprros _............... 325

Diphÿlleia _............_._._. 293

Diplachne _................ 356

Dipsacus _...................... $3 \mathrm{I} 4$

Dirca -..._- 337

Discopleura _..._.............. 311

Dodecatheon _.................... 325

Draba _............ 295

Drosera _.............. 293

Dulichium _................ 349

Eatonia _._.

Echinacea_._._._._._._._. 320

Echinodorms _.................... 342

Echiun1 -._._._._. 332

Eiclipta_._._.

Eleocharis _._._._._._._... 349

Elephantopus _._._............ 315

Eleusine -................... 356

Elodea _..._................ 343

Elodes _........._. 299

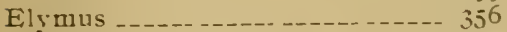

Epigaea _... . . - .

Epilobium -................ 310

Epiphegus _..._._._._._. 326

Equisetum _..._._.......... 357

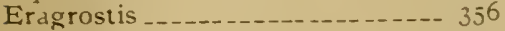

Erechthites_..._._.

E:ianthus _.................. 352

Erigenia _............ 312

Erigerou _................... 318

Eriocaulon _................... $3+\mathrm{S}$

Eriophorum ................ 349

Erodium _... 300
Errngium ...............

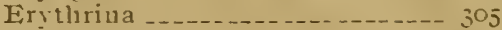

Erythronium -................. 345

Euouy mins _................ 301

Eupatorium _..._............. 316

Euphorbia _.................... $33 \mathrm{~S}$

Fagopyrum _... . . .

Fagus _...... - 339

Festuca -.....

Filago -...

Fimbristylis _................ 349

Foeniculuu _................. 314

Fothergilla _................. 309

Fragaria - _..._. 306

Frasera - .

Fraxinus _._.

Fuirena _..._. 349

Fumaria _............. 294

Galactia ............... 305

Galax _..................... 324

Galeopsis _................. 333

Galium _..._.................. 313

Gallinsoga .................... 32 I

Gaultheria _.................. 324

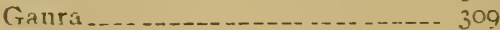

Gavlussacia ................ 324

Gelsemium ..................... 314

Gentiana ..._._._._.......... 329

Geranium -................... 300

Gerardia _................... 327

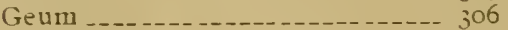

Gilleuia _..._.

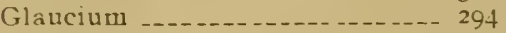

Gleditchia ..................... 305

Glyceria _._._._._. 354

Graphalium _................. 319

Gonobolus _................. 33 I

Goodyera ................... $3+3$

Gordonia . _._._.

Gratiola ...................... 327

Gyulnopogon_................. 356

Gyuandropsis ................ 295

Habenaria _._. . . _ _._._. 343

Halesia _..................... 325

Hamamelis ............... 309

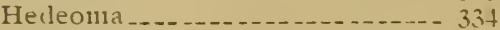

Helenium _................ $32 \mathrm{I}$

Helianthemum ................ 296

Helianthus _._.

Heliopsis _.................. 320

Heliotropium ................ 33 I

Helleborus _.................. 292

Hewerocaulis ............... 346

Hemicarpha . . . . . . . . . . 349

Hepatica .................... 29 I

Heracleum _................. 312

Herpestris ................... 327

Hesperis -.................. 295

Heteranthera ................... 347

Heuchera _.................. $30 \mathrm{OS}$

Hibiscus _.................... $29 \mathrm{~S}$ 
Hieracum PAGE.

Hierocbloe

Holcus _.................... 351

Hordend ................... $3: 5$

Hosacisia................... 303

Hotin дia _................ 325

Houstovia................... 313

Hudsonia .................. 2c5

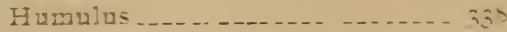

Hrdzargea .....

Yirdrastis ................ 2, 2

Hrdtochloa ................... $3=4$

Hritrocots]e................. 3. II

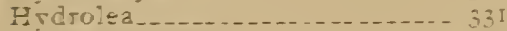

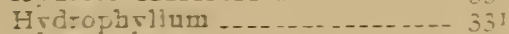

Hrmenacallis ..... ........... 344

Hгрericur ............... - 290

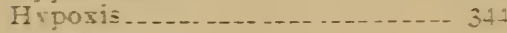

HiF215

Hvssopus ................. 331

I

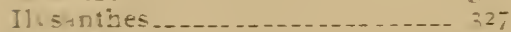

Im] patuevs ................... $3 x$

Indi zofera ............... 503

Iтvia ...

ipgmeé _.................. 25

Iros . .

I<antious . .

I=riepis _...

I I iprrim

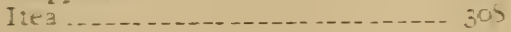

Iหล ....

Ixia

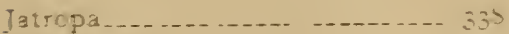

jeİersovja ................ 293

Juylans . . . . . .

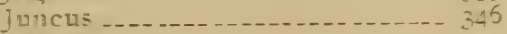

juziperus ................

jussjaea

Ju-ijcia . . . .

Kalumia_f.................. 323

Sosteleizłra ............... 29s

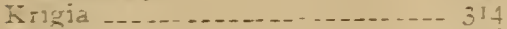

Euhria .......................... 316

K, lingia ............ 319

Lachiauthes _... ...

L.chuocaulov ................ 34)

Lactuca _.................... 314

Lagezaria ............ 310

Lagium .................... 333

Laporiea _.. . . . . . .

Lappula _................ 331

I.athrrus . ... ................. 304

Lecbea _.................... 295

Leetsia _.................. 354

Leicphrllum ............... 325

Lexna ...................... 342

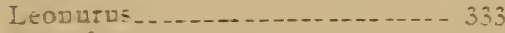

Lepacbss ....

Lepidiug] ................... 295
PAGE.

Leptocaulis

Leptochloa .................. 356

Lespedeza _.................... 304

Leucotboe................... 327

Liattis _....................... 315

Ljgusticum .................... 31 I

Lilium ................... 346

Limралthemun ............ 330

Linnobium .................. 313

Linaria..................... 325

Lindera _.................. 337

Livum ................... 299

Liparis _............... 314

Lipocarsba _................. 349

Lippia ....

Liquidamber ................. 309

Liriorendton ................. 203

Lictera _...............

Lithospermuan ................ 3jI

Liisea ...

Lohelia

Lolium _...................... $\$ 55$

Lonicers .................. $5 ! 3$

Lophanthus ................. 333

Loplijola ................... 311

Lotus ........................ 503

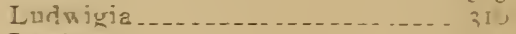

Lนріจนร _._. _...

Lนzน] 1....................

Lichnis _.................... 297

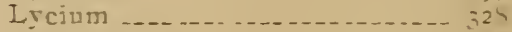

Lịcnpodium ................. 35

Lycopus .... . . . . . . . . 334

LTcopsis ................... 332

Lsgodium .............. 355

Lrsim achia ................. 325

Lithrum _.............. 309

Macbridea...... . .

Magnolia_._............... 292

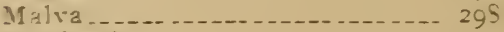

Manisuris _................... 352

Marrutiam................ 333

Marshallia _................. 321

Bart 1ria_._ _._. 325

Maraca _... ............ 347

Mediola _.................. 375

Merlicago _.................. 303

Melamprrum ................. _ 325

Melantbium ..................... 345

Melia __ _...

Melica _............. 356

Melilotus................ 302

Melissa_.................. 334

Melothria -................ 310

Merispermum _._._._._._._. 293

Mentba -......

Menziesia _............... 323

Mertensia .................. $3.3 \mathrm{I}$

Micrantbemum ............... 327

Microstylis ................. 313

Mikania_..................... 316

Milium -_. 


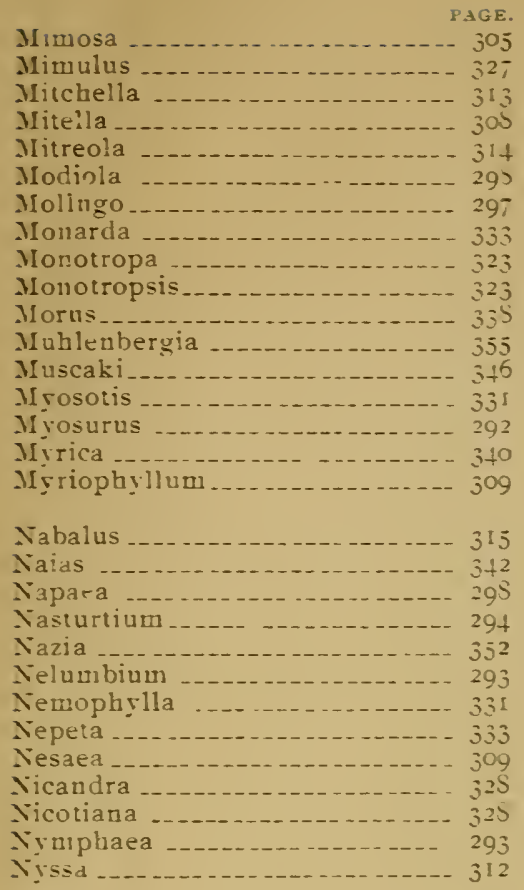

Obolaria _........

Ocimum .................... 332

Ennthera ................... 310

O:den?andia .................. 313

Onoclea ..................... 353

Onosmodium .............. 332

Opuntia _................... 31

O:chis ................... 343

Orrganum _.................. 334

Ornithogalum _............. 346

Osmanthus ................... 331

Osmorhiza ................. 312

Osmunda _...................... $35 \mathrm{~S}$

Ostrya _...

Otophylla _.............. 327

Oxalis _............... 299

Oxicoceus ................. 324

Oxilendrum .................. 324

Pachrstima................... $30 \mathrm{r}$

Palpalanthus ................. 3t

Pailax _............. 312

Panicum -...

Papdver ...

Pdrietaria _....................... 3.

Parnassia ................. so.

Paronychia ................ 297

Parthénium ............... 320

Paspalum ..................... 352

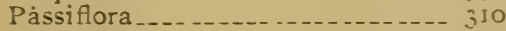

Pastinaca_.................. 312

Paulownia _................... 327
Pedicularis ............. $32 S$

Peliaea

Peltandra .................. $34 \mathrm{I}$

Penthorium _._-_._-_._..... 309

Penstemon _.................. 326

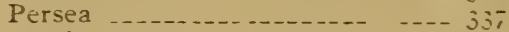

Petalostemon -.............. 303

Phacelia _.................. $33 \mathrm{I}$

Phaiaris .

Phaseulus _..._ ............... 305

Phegopteris ................. $355^{3}$

Philadelphis ................. 305

Phleum _..................... 354

Phlox _... - .

Phorodendron _............... 337

Phragmites................... 356

Phryma -. ... .

Phịllauthus ................. 335

Phrsalis .................... 325

Physostegia .......... 333

Plirtolacca ............. 335

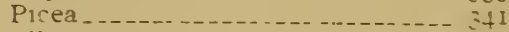

Pilea _.......

Pimpermella _......... 311

Pinguicula _................ 326

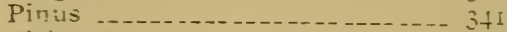

Pirique:a .................... 310

Planera -........... 339

Plantago _.......... 335

Piatdnus .................. 339

Pleea _............... 346

Pluchea _.............. 319

Рон ....

Podophyllum ................. 293

Purlostemoli _................ 337

Polostugma.............

Pogonia _................... $3 \div 3$

Pulanisia _................... 295

Poiemuniun............... 329

Polıgala

Polrgonatum ................ 345

Poil gonella ................. 336

Polygontinl.... _... 330

Poly unia _...... ... 320

Polypodium _ …

Polypogon.... _... _... 355

Polypremum _ . ... .. . 314

Pontederia ... . . . . . .

Ponthieva ......... _ 344

Populus . . . . . . . . . . $\quad 340$

Portulaca..... ..... ..... 29.

Putamogeion ... ... .... 342

Potentilla .... . . . . . . . . 306

Prosartes . ... . . . . . ... 340

Pruserpinaca - .... . . . . . 309

Prunella .... . . . . . . . . . . 333

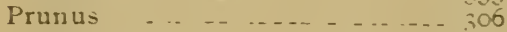

Psilucarya _.....

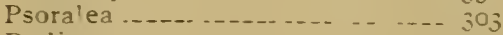

Ptelia _......

Pteris -..... 357

Pterocaulon ................. 319

Pycnanthemum ................. 334

Prrola ........ 323 


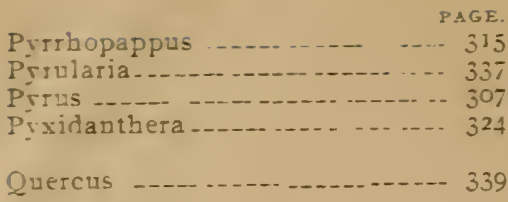

Ranunculus ............. 291

Raphanus _.................. 295

Rhanมน ..................... 30I

Rhesia ... ......... ... . . 309

Rhododendron ............. 323

Rhus . . . ... . . . . . . . 300

Rhruchosia ................... 305

Rbinchospora .............. ... 349

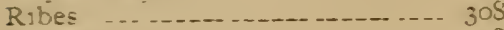

Ricinus ........................... $33 \mathrm{~S}$

Robinia ... ............... 303

Rusa ........................ 307

Rubus ....... .................. 306

Rudbeckia ................ 320

Ruellia _............... 332

Rumex ..... .............. 336

Ruppia ..................... 342

Sabal ...................... $34 \mathrm{I}$

Sabbatia _... . .

Sagina - ... . . . 297

Sagittaria_..... ......... . 342

Salicornia ................. 336

Salix .... ................ 340

Silsola ............... 336

Salvia .................... 333

Sambucus -................ $3^{12}$

Samolus ...................... 325

Sanguinaria ...... -........ .. 294

Sanguisorba -............... 305

Sanicula..... ............. 3 II

Siponaria -..

Sarracenia ..... ............ 293

Sassafras _ .......... - .. 337

Satureia ................ 334

Saururus ...... -.......... 337

Saxifraga -................ 30 ob

Scandix _.................. 312

Schrapkia .................. 305

Schwalbea ................... 327

Scirpus ..... -... ... ... - 379

Scleranthus -..... -........... 295

Sclevia -..................... 350

Sclerolepis .... ........... - $\quad 3^{1} 5$

Scrophularia ... ....... -. 326

Scutellaria ................ 333

Serlum ................ 308

Selaginella ............... 358

Senebiera................... 295

Serecio .................... 322

Sentera -................. 330

Sericocarpus................ ... 316

Sesbauia _.................. 303

Sesurium _.................... 311

Setaria ............... 354

Sermeria .................. 327

Shortia _..................... 324
Sicyos PAC

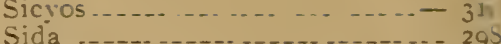

Silene ...

Silphiun ............ . . 320

Sinapis _. . . . . . 295

Sisymbrium -................ 295

Sisrrhinchium ............... 344

Sium .... .................. 3 II

Smilacena -...... - .

Sinilax . . . . . .............. 345

Solazum _............. 325

Solea .... . .

Solidago..................... 315

Sonchus -.... ............. 314

Sorghum .............. - 352

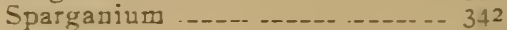

Spartina .. - ....... -... 356

Specularia ...... ...... . . ..... 323

Spergula _............... 297

Spigelia ................ 314

Spilanthes ... . . . . . . . . .... 320

Spiraea .... - ............ 305

Spiranthes -............... .. 343

Sporobolus ....... - ... - - 355

Stachrs .... -................ 333

Staphrlea -.. . . . .

Statice -... ........... 325

Steironema ................. 325

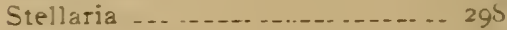

Stenanthium ............ 346

Stillngia _... - .

Stipa

Stipulicida ..... ........... 299

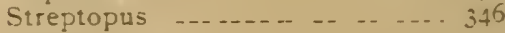

Stuartia ... . . . . . . . . . . 299

Stylosauthes _.. ...... - . . ... 304

Strrax ..... . . . . . . . . . 32.5

Suaeda _............. . 336

Srmphoricarpus ...... ......... 313

Siniplocus . ............. 325

Srmphrtum -........... 3.32

Sinindra ... . . . .......... 333

Syringa ............... -... $33^{1}$

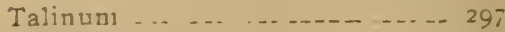

Tanacetum ...... . . . . . 322

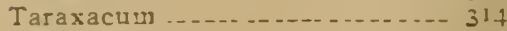

Tasorium ................ 341

Taxus .... ... ... ...... ... 341

Tecoma ................. 326

Teprosia -................. 303

Tetragnnotheca ...... ........ 320

Teucrium -... -.......... 332

Thalictrum .............. 291

Thaspium _................. 311

Thermopsis ................... 305

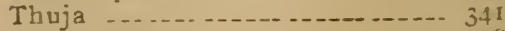

Tiarella _.. ............ 308

Tiedmauria ................. 312

Tilia ... - .... . . . . . . . ... 29.5

Tillandsia .................. 344

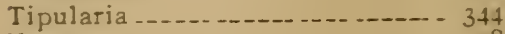

Tissa ........................ $29 \mathrm{~S}$

Tofieldia .... ......... 346 
PAGE.

Idescantia -..... -.. . . ... 34t

ragia -.. -..._-...... - . - 335

ragopogou .... ..... .. ... .. 314

irautvetteria _.............. 29 t

Trichostema ...

Trifolium -....

Iriglochin ............... 343

Trillium …

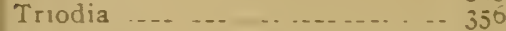

Triosteum ....... _......... $\$ 13$

Triplacis ............... 350

Tripsacum …

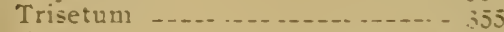

Tsuga ..... ............ $3+1$

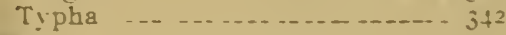

¿lmus ... . . . . . . . . . . 339

Eniola ..... . . . . . 356

C'rtica.....

Liricularia ........... ... - 325

Evularia -... $\quad 345$

Vaccinium -........ . . 324

Valeriana .....

Valerianella ... . ............ 314

vallisceria . . .............. - 343

Veratrum ...... ....... $3 \$ 6$

Verbascum ................ 326

Verbeua..... -... . . . . . . 332

Terbesina .... .............. 32 !
PAGE.

Vernonia .. - . . . . . . 315

Veronica ...................... 327

Viburnum ..................... $3^{1} 3$

Vicia . . .

Vigua -..... ...

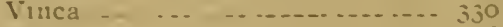

Vivla ...

Vitis ....................... jor

W'al Isteinia _.

Wistaria .... ............. \$03

IVolffia ................ 315

Wuodsia ….... ... - 350

lloodwardia ...... . . . .

Cautbiuu .................... 315

Xerophyllum. .............. 346

Xiris ... . . . . . .... 347

Yucca . . .

Zauthorhiza ...... . . .

Zanthoxylum

Zaunichellia _..... . .

Zınuia ... ............... 320

Z:zania - .............. 354

Zizia ............... 311

Zorvia .... ......... ... 304

Zostera -................. 342

$Z_{1}$ gademus ................. 346 

